\title{
Valuation, Investment and the Pure Profit Share
}

\author{
Pierre Lafourcade * \\ Board of Governors of the Federal Reserve System
}

November 14, 2003

\begin{abstract}
This paper explores some implications for valuation and investment of challenging the standard assumption that there are no aggregate pure profits in the US economy. First, it highlights the theoretical importance of monopoly rents for fluctuations in average $Q$. A series for such rents is then computed by assuming that production is Cobb-Douglas, as fluctuations in the output share of pure profits may be inferred from variations in the labor share. Consequently, the paper focuses on the correlation between a measure of rents and observable average $Q$. It also reassesses the empirical disconnection between investment and a measure of marginal $q$ purged of monopoly rents. The paper finds that the existence of pure profits as constructed from unit labor costs only accounts for about $5 \%$ of fluctuations in observed $Q$, and alters only minimally the empirical relationship between $q$ and investment.
\end{abstract}

JEL Classification: G00, E20, C50

Keywords: Tobin's $q$, investment, markup, scale, rents

*Address: Federal Reserve Board, Washington, DC, 20551. E-mail:pierre.lafourcade@frb.gov. The author would like to thank Jason Cummins, Stacey Tevlin, Peter Tinsley and Stephen Wright for helpful comments. The views expressed in this paper are solely the author's and do not necessarily represent those of the Federal Reserve Board or its staff. 


\section{Introduction}

The purpose of this paper is to construct and analyse the properties of a series for Tobin's $q$ that acknowledges the presence of pure profits. More specifically, this paper attempts to quantify the Hayashi (1982) decomposition of 'average' $Q$, the observable ratio of the value of the firm to its capital base, as

$$
\frac{V}{K} \equiv Q=q+q^{\pi}
$$

where the first term on the right hand-side is 'marginal' $q$, and the second term is the present discounted value of monopoly profits. The importance of this decomposition is brought to bear when considering the cornerstone of the $q$ theory of investment, namely that it is unobservable marginal $q$, and not average $Q$, which is a sufficient statistic for investment.

The vast empirical literature on investment proposes a number of explanations for the empirical failure of average $Q$ to account for investment fluctuations: capital market imperfections (Fazzari, Hubbard and Petersen, 1988), non-convex costs of capital adjustment (Caballero, 1999), noise in share prices (Bond and Cummins, 2001), gross mis-measurement of true capital (Hall, 2001). This paper documents another possible reason for the lack of valuation-investment correlations that appears to have received little attention: the presence of rents which accrue to firms with market power.

To motivate this point, it is useful to return to Hayashi's (1982) seminal contribution to the empirics of investment behavior. Hayashi showed that, under certain conditions discussed below, the unobservable value of a marginal unit of a firm's capital is equal to the observable value of its average unit. This result warranted the substitution of average $Q$ for marginal $q$ on the right-hand side of investment equations.

Hayashi also showed that relaxing the assumptions underlying this equivalence result generates a wedge between average and marginal $q$ which may capture a number of features: putty-clay technology, the nature of the tax system, non-constant returns in production, or rents accruing from market power. Although the first two features are certainly empirically relevant, this paper focuses exclusively on the interplay between markup and scale which generates the pure profits term $q^{\pi}$ in equation (1). In this instance, firms are valued not 
only on the basis of the marginal unit of capital that they own (captured by marginal $q$ ), but also on the present value of the profits that they extract from their monopoly positions $\left(q^{\pi}\right)$. These supernormal profits are interpreted as revenues that a firm obtains over and above all opportunity costs, including in particular a market-determined rate of return on capital. They are rents over whatever property right confers the monopoly power. Property rights are used in the largest sense, including ownership of land, brand names, public reputations, franchises, patents, all characteristics that allow the owner of the rights to create and perpetuate monopoly power.

This paper follows the methodology developed by Abel and Blanchard (1986). To sidestep Hayashi's conditions for substitution of average for marginal $q$, these authors make assumptions about the profit function of firms to construct directly a measure for marginal $q$. They then investigate whether this computed series is more successfully correlated with investment than average $Q$. Similarly, this paper computes a measure of marginal $q$, but the analysis accounts for a factor which Abel and Blanchard overlooked, namely the possible existence of rents.

In effect, these authors conjectured that firms are price-takers and that the underlying production function is homogeneous of degree one in inputs, implying that the average profit of capital is equal to its marginal profit. The consequence of these assumptions is that, with reference to equation (1), the authors implicitly build a constructed measure of $q+q^{\pi}$ instead of computing a series for $q$. That is, they do not differentiate between the returns that arise from the productive use of an extra unit of capital from those that accrue only from the market position of the firm. Yet equation (1) shows that this can be misleading for the purpose of inference about the correlation between valuation and capital accumulation, as marginal $q$ is the sole driver of investment. In particular, Lafourcade (2003a) showed in a calibrated general equilibrium model that this wedge between average and marginal $q$ could theoretically cause substantial swings in valuation which are indeed uncorrelated with investment.

As was mentioned in the paragraph before last, this wedge depends on firms' pricing power and cost functions. Crucially, however, although both the markup and elasticity of scale are variables of interest in themselves, it is only their ratio that matters for determining $q^{\pi}$. This 
paper's leverage comes from observing that under the standard assumption of Cobb-Douglas production, their ratio fluctuates inversely with the labor share. This implies that variations in $q^{\pi}$ can be quantified, and inferred from the sole labor margin, independently of hypotheses about adjustment costs on the capital margin.

Given this simple observation, the paper is organized with two issues in mind: the relationship between average $Q$ and rents, and the link between investment and a rent-free measure of marginal $q$.

Section 2 argues that average $Q$ can fluctuate for reasons independent of capital adjustment costs. In particular, it puts forward two possible explanations that may potentially overlap: the existence of pure rents and the presence of intangible capital. The relative merits of these reasons are analysed theoretically. The rest of the paper, however, focuses exclusively on the rents channel.

Section 3 constructs sample paths of $q^{\pi}$ by exploiting the congruence of variations in the pure profit share with those of the labor share. The decomposition (1) is then used to investigate the extent to which observed fluctuations in average $Q$ are attributable to those in $q^{\pi}$. Since $q^{\pi}$ captures expectations of future monopoly profits, one must estimate the timeseries properties of the information set over which these expectations are formed. The section considers both a univariate and a vector-autoregressive (VAR) approach to this computation. The main result is that fluctuations in rents are significant and relatively strongly correlated to observed average $Q$, but that these variations contribute only marginally - around $5 \%$ - to fluctuations in observed average $Q$.

Section 4 investigates the impact of accounting for monopoly rents on Abel and Blanchard's (1986) analysis of the correlation between marginal $q$ and investment. Similarly, this rents-based extension to the $q$ theory adds very little explanatory power to the relationship between valuation and investment. The final section concludes. 


\section{Asset prices and rents}

\subsection{Campbell and Shiller (1988) revisited}

Consider the workhorse of asset pricing models, the stochastic pricing equation:

$$
1=E_{t} R_{t, t+1} P_{t+1}
$$

where $P_{t+1}$ and $R_{t, t+1}$ are the gross return obtained from holding an asset between periods $t$ and $t+1$, and a stochastic discount factor used for pricing assets for that period, respectively. Applied to equity holdings, this pricing equation yields the standard arbitrage equation

$$
1=E_{t} R_{t, t+1} \frac{V_{t+1}+D_{t+1}}{V_{t}},
$$

where $D_{t}$ is the dividend the firm pays to its stockholders and $V_{t}$ is the ex-dividend value of the firm.

This equation can be used to analyse a number of financial indicators, grouped into priceto-flow and price-to-stock ratios. In a landmark paper, Campbell and Shiller (1988) explore the informational content of an element of the first group, the price-dividend ratio $V / D$. By log-linearising the equation, forwarding it and imposing a transversality condition, they show how a high ratio captures expectations of high future dividend growth or low returns. Cochrane (1997) provides a thorough review of the ensuing literature that concentrates on price-dividend ratios to forecast returns.

The same methodology can be applied for a member of the second group, average $Q$, whose relationship with the price-dividend ratio is readily seen by splitting the latter into two components:

$$
\frac{V_{t}}{D_{t}} \equiv \frac{V_{t}}{K_{t+1}} \frac{K_{t+1}}{D_{t}}
$$

The price-to-flow dynamics of the price-dividend ratio are captured by average $Q \equiv \frac{V_{t}}{K_{t+1}}$ and the stock-to-flow dynamics of the (inverse of the) payout rate - what Abel and Blanchard (1986) call the average profit rate. In two recent papers, Robertson and Wright (2002a, 2002b) analyse the dynamic log-linear version of this identity in detail, in the spirit of Campbell and Shiller. Where the price-dividend ratio predicts future dividend growth or returns, 
average $Q$ should theoretically predict future investment (capital growth, in other terms), returns and payouts (which captures profitability).

Both frameworks focus on forecasting returns in the long-run, and take dividends to be an observable time-series process. However, going further upstream and making explicit assumptions about the general equilibrium process for dividends may arguably shed some light on the behavior of asset prices and their relationship to fundamentals. ${ }^{1}$

In the usual formulation, dividends are defined as the residual of output-importantly, a value-added measure - after investment and labor are paid for. Under the assumption of perfect competition and constant returns to scale in both product and factor markets, value-added is exhausted by payments to inputs

$$
Y_{t}=r_{t} K_{t}+w_{t} N_{t}
$$

where $w$ and $r$ are the competitive real wage and rental price of capital, respectively. Since investment is the change in capital net of depreciation, ${ }^{2}$

$$
I_{t}=K_{t+1}-(1-\delta) K_{t}
$$

dividends are

$$
\begin{aligned}
D_{t} & =Y_{t}-w_{t} N_{t}-I_{t} \\
& =\left(r_{t}+(1-\delta)\right) K_{t}-K_{t+1},
\end{aligned}
$$

\footnotetext{
${ }^{1}$ Note that the current paper has nothing to say about debt, taxation or equity buybacks.

${ }^{2}$ The timing assumption is the following. Uncertainty arises from the nature of the income generating process $Y_{t}$, which is subjected to stochastic technology shocks or demand disturbances at the beginning of period $t$, before time $t$ allocations are made. $K_{t+1}$ is the capital stock at the close of period $t$, available for production at the start of period $t+1$. Because investment $I_{t}$ is chosen at the beginning of period $t, K_{t+1}$ is known at that same moment. This implies that the dividend process $D_{t}$ is also known at the beginning of the period. Thus capital $K_{t+1}$, output $Y_{t}$, consumption $C_{t}$, investment $I_{t}$, labor $N_{t}$ and dividend $D_{t}$ are all part of the information set available at the end of period $t$ over which expectations of future variables are computed. The current framework is demand-driven, with no idle capacity. The implication for equation (3) is that both $V_{t}$ and $D_{t}$ are part of the information set over which expectations are computed, but $V_{t+1}$ and $R_{t, t+1}$ are not.
} 
where $\delta$ is the depreciation rate. ${ }^{3}$ Replace in the valuation equation (3) to obtain

$$
1=E_{t} R_{t, t+1} \frac{V_{t+1}+\left(r_{t+1}+(1-\delta)\right) K_{t+1}-K_{t+2}}{V_{t}}
$$

Multiplying both sides by average $Q$, and using the stochastic pricing condition (2) for capital, whose expected return is $r_{t+1}+(1-\delta)$, yields the following:

$$
\begin{aligned}
\frac{V_{t}}{K_{t+1}} & =E_{t} R_{t, t+1} \frac{V_{t+1}+\left(r_{t+1}+(1-\delta)\right) K_{t+1}-K_{t+2}}{K_{t+1}} \\
\frac{V_{t}}{K_{t+1}}-1 & =E_{t} R_{t, t+1} \frac{K_{t+2}}{K_{t+1}}\left(\frac{V_{t+1}}{K_{t+2}}-1\right) \\
Q_{t}-1 & =E_{t} R_{t, t+1} G_{t+1, t+2}\left(Q_{t+1}-1\right),
\end{aligned}
$$

where $G_{t, t+k} \equiv \frac{K_{t+k}}{K_{t}}$ is the $k$-period compound growth rate of capital. Clearly, the only solution to this equation is $Q_{t}=1$ for all $t$, which means that $V=K$. This is the standard result for valuation in the perfect competition-constant returns to scale framework with no adjustment costs: the value of the firm is equal to its capital base at every point in time.

\section{$2.2 \quad$ Average $Q$ and the pure profit share}

As is well-known, the neoclassical model's explanatory power for asset price volatility is particularly poor, insofar as capital movements are much smoother than fluctuations in valuation. This failure is usually explained by the absence of variation in the price of capital, since installed and uninstalled capital are perfect substitutes in producing new capital. The usual way of generating fluctuations in this price is to introduce installation costs.

This subsection, however, suggests another source of fluctuations in average $Q$, one which stems from pricing power. Return to the arbitrage equation (3). Suppose that market power prevails in product markets concurrently with increasing returns to scale. Factor markets are still taken to be perfectly competitive, and the production function is assumed to be

\footnotetext{
${ }^{3}$ Note that the present definition of dividends differs from Abel and Blanchard's (1986), where their operational measure of dividends - or 'profits' in their terminology — is output net of the wage bill only. This implies that their profit function is linear homogeneous in capital alone, which is inconsistent with the standard structure of $q$ models (see Bond and Cummins (2001)). For comparative purposes, Section 4 will refer back to their measure. For the rest of the paper, however, dividends are defined net of investment costs.
} 
homogeneous in capital and labor:

$$
F(K, N)=F_{K} K+F_{N} N
$$

Firms with market power reward inputs with less than their marginal products:

$$
F(K, N)=\mu(r K+w N)
$$

where $\mu$ is the ratio of the price of value-added to marginal cost. However, in the presence of fixed costs $\Phi$, value-added is $Y=F-\Phi=\frac{1}{\eta} F$, where $\eta=F /(F-\Phi)$ is a local measure of elasticity of scale. Therefore,

$$
\eta Y=\mu(r K+w N)
$$

This shows that unless the scale parameter is equal to the prevailing markup, pure profits $\Pi=\left(1-\eta \mu^{-1}\right) Y$ are possible ${ }^{4}$. Writing the gross pure profit share as $\pi_{t} \equiv \mu \eta^{-1}=\frac{Y}{w N+r K}$, dividends are

$$
\begin{aligned}
D_{t} & =Y_{t}-w_{t} N_{t}-I_{t} \\
& =\left(\pi_{t}-1\right)\left(r_{t} K_{t}+w_{t} N_{t}\right)+\left(r_{t}+(1-\delta)\right) K_{t}-K_{t+1} \\
& =\left(1-\pi_{t}^{-1}\right) Y_{t}+\left(r_{t}+(1-\delta)\right) K_{t}-K_{t+1} .
\end{aligned}
$$

Dividends are now composed of two revenue flows: one arising from the competitive rental market for capital, and the other - the first term on the right-hand side of (5) - generated by an existing (possibly temporary) monopoly franchise in output markets. Replacing (5) in the arbitrage equation (3) produces the following:

$$
1=E_{t} R_{t, t+1} \frac{V_{t+1}+\left(1-\pi_{t}^{-1}\right) Y_{t+1}+\left(r_{t+1}+(1-\delta)\right) K_{t+1}-K_{t+2}}{V_{t}}
$$

\footnotetext{
${ }^{4}$ Note that the same result is obtained when returns to scale are introduced directly by assuming that the production function is homogeneous of degree $\eta>1$, so that

$$
\eta F(K, L)=F_{K} K+F_{N} N
$$
}


and equation (4) becomes

$$
Q_{t}-1=E_{t} R_{t, t+1} G_{t+1, t+2}\left(Q_{t+1}-1\right)+E_{t} R_{t, t+1}\left(1-\pi_{t+1}^{-1}\right) \frac{Y_{t+1}}{K_{t+1}}
$$

Average $Q$ can now fluctuate because of expectations of future monopoly profits.

As was mentioned previously, Hayashi derived an equivalent result. Yet the bulk of the ensuing literature failed to test the empirical relevance of this extra term, either by assuming away these pure profits or failing to differentiate them from the competitive return to capital. A number of authors have argued that there are no rents to be exploited in the long run in the US economy, as average $Q$ is equal to one on average over the post-war period (Rotemberg and Woodford, 1995). Regardless of how empirically controversial this statement is (Robertson and Wright, 2002a), this does not preclude short-run pure profits. Assuming that entry-andexit dynamics erode any existing pure profits in the long-run indeed imposes the restriction that $\mu=\eta$ and hence that $Q=q=1$. However, in the short run, $Q$ can still deviate from its steady-state value if some temporary pure profits are expected in the future ${ }^{5}$. The magnitude and the duration of this deviation will depend on the initial size of the extra profits generated and the speed of subsequent entry, but entry need not be instantaneous. Consequently, these deviations come over and above those that would be induced by the presence of adjustment costs, and a fortiori fluctuations in investment. Because this measure of $Q$ is solely dependent on rents, it is renamed $q^{\pi}$ (the superscript recalling the pure profit share). It is the same term as that on the right-hand side of equation (1).

Taking a first-order Taylor-expansion around the steady-state rates of return and growth $R, G$ and around $\pi=\mu \eta^{-1}=1$ and $q^{\pi}+1=1$ (with deviations from the steady-state noted with a 'hat') yields:

$$
\hat{q}_{t}^{\pi}=R G E_{t} \hat{q}_{t+1}^{\pi}+R \frac{Y}{K} E_{t} \hat{\pi}_{t+1}
$$

\footnotetext{
${ }^{5}$ The equations in this section should be seen as the short-hand aggregation of symmetric firms producing differentiated goods indexed $i \in[0, n]$ (see Rotemberg and Woodford (1995) or Lafourcade (2003a)). Each is faced with the same elasticity of demand and same sunk costs $\phi$ of producing at each point in time. Thus an entrant with a new product earns the same profits as the producer of an existing good. Therefore, the presence of sustained pure profits in existing markets induces entry in new markets, thereby increasing $n$, aggregate fixed costs $\Phi=\int_{0}^{n} \phi d i$, and decreasing aggregate profits for a given use of aggregate inputs. Sustained pure profits are generated from technology or markup shocks.
} 
From the firm's optimality condition on capital,

$$
r=(1-\alpha) \frac{Y}{K}
$$

where $(1-\alpha)$ is the elasticity of output with respect to capital. The equilibrium, utility-based stochastic discount factor is

$$
R=\beta G^{-\gamma}=(r+(1-\delta))^{-1}
$$

where $\gamma$ is the coefficient of risk-aversion. Forwarding the previous expression, replacing terms and assuming the relevant transversality condition holds, yields

$$
\hat{q}_{t}^{\pi}=\frac{r R}{(1-\alpha) \rho} \sum_{i=1}^{\infty} \rho^{i} E_{t} \hat{\pi}_{t+i},
$$

where $\rho=G R<1$ for the relevant sum to converge.

To the extent that pure profits are autoregressive, which they should intuitively be if entry eliminates them in the long run, expected profits may be predicted by current profits. ${ }^{6}$ Some interesting statistical properties arise from the discounted-sum formulation of this rents-based measure of average $Q$, features reminiscent of the effect of the persistence of expected asset returns on current asset prices discussed in Campbell, Lo and Mackinlay (1997, Chap. 7). Mirroring their analysis, suppose that the expected pure profit share equals a zero-mean variable which follows an $\mathrm{AR}(1)$ process:

$$
E_{t} \hat{\pi}_{t+1} \equiv \hat{\pi}_{t}^{e}=\phi \hat{\pi}_{t-1}^{e}+\varepsilon_{t}
$$

This implies:

$$
\hat{q}_{t}^{\pi}=\frac{r R}{1-\alpha} \frac{1}{1-\phi \rho} \hat{\pi}_{t}^{e}
$$

This equation gives the effect on average $Q$ of variations through time in the expected pure

\footnotetext{
${ }^{6}$ As a flipside to this argument, it also seems intuitive that entry is rational only insofar as profits are predictable.
} 


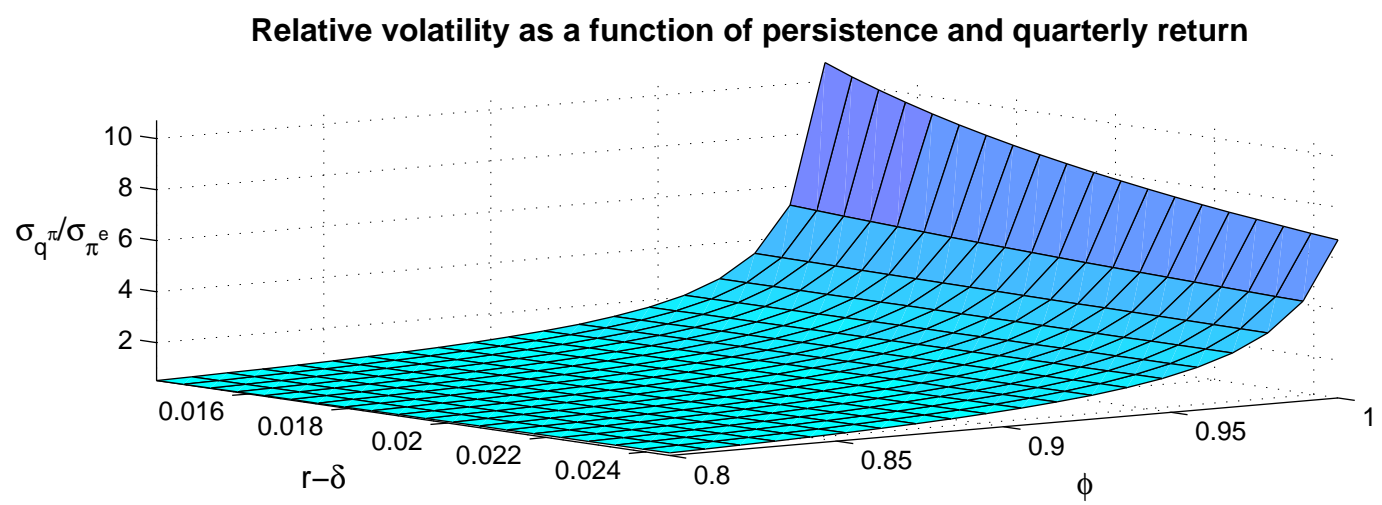

Relative volatility as a function of persistence and quarterly growth

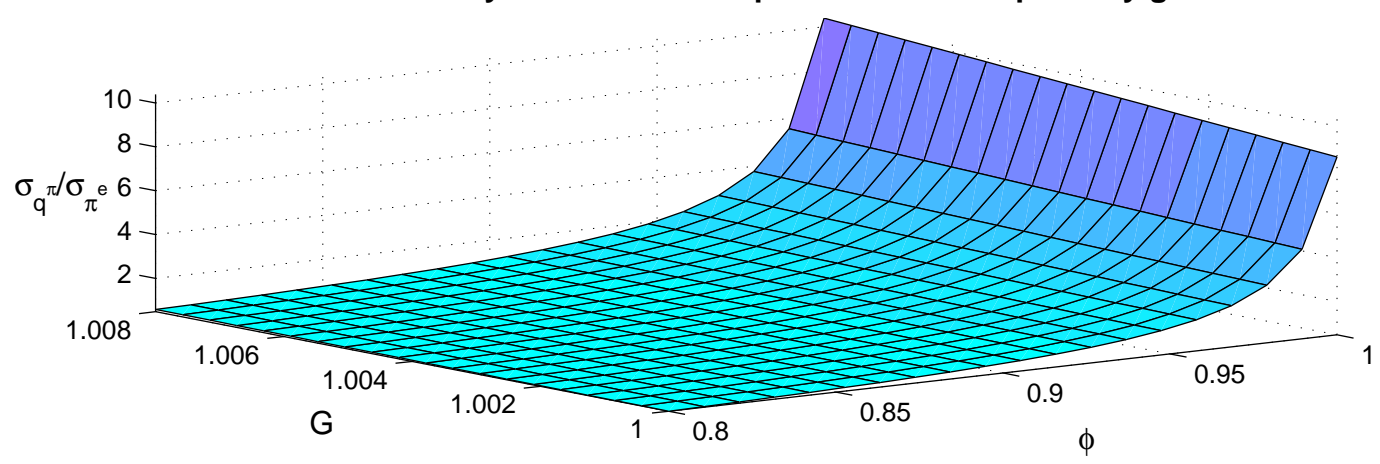

Figure 1: Sensitivity analysis

profit rate. That is,

$$
\frac{\sigma_{q^{\pi}}}{\sigma_{\pi^{e}}}=\frac{r R}{1-\alpha} \frac{1}{1-\phi \rho}
$$

where $\sigma_{z}$ is the standard deviation of $z$. A change in the expected pure profit rate has a greater effect on average $Q$ when it is persistent.

A simple calibration gives a feel for the magnitude of this effect. Take the quarterly values that Rotemberg and Woodford $(1995,1996)$ use:

$$
\begin{array}{|l|l|l|l|l|}
\hline \alpha=0.64 & r=0.041 & \delta=0.025 & \gamma=1 & G=1.004 \\
\hline
\end{array}
$$

A $1 \%$ increase in the expected profit rate from its equilibrium value of 1 increases $q$ by $0.36 \%$ if $\phi=0.7$, but by $1 \%$ for $\phi=0.9$, and $3.5 \%$ for $\phi=0.98$ (a value implicitly used by Rotemberg and Woodford (1995)). In the limiting case where expectations are not mean reverting, i.e. $\phi=1$ (a situation that is somewhat difficult to reconcile with the assumption of free entry), these parameters would lead to an almost tenfold response of $q^{\pi}$ for any given change in $\hat{\pi}^{e}$. 
Figure 1 traces the ratio of the standard deviations of $q^{\pi}$ and $\hat{\pi}^{e}$ as a function of the steady-state quarterly real rate of return $r$, the steady-state growth rate of technology $G$ and the persistence parameter $\phi$. Because $G, \beta$ and $R$ are pinned down by the steady-state Euler equation (8), the first plot assumes that $G=1.004$ while the subjective discount factor $\beta$ varies to generate the observed values of $R$. Similarly, the second plot assumes $\beta=1.004 / 1.016$ while $G$ takes on different values, and $R$ varies accordingly. Note the magnifying effect $\phi$ has on the ratio of volatilities over the range of values of rates of return and growth which are consistent with observed data. The parameter $\phi$ increases the sensitivity of asset prices to the choices of underlying structural parameters such as the discount rate and technology growth. This result motivates the next step in this paper: estimating the data-generating process for rents, to obtain $\sigma_{\pi^{e}}$ and $\phi$.

\subsection{Average $Q$ and intangibles}

Before estimating the pure profit share, however, it is useful to compare the model developed in this paper with a recent competing model of Tobin's $q$.

Capital, the denominator of average $Q$, is usually taken to be the stock of tangible assets of the firm (see, for example, Blanchard, Rhee and Summers (1993), Robertson and Wright (2002a)). To be consistent with this definition, marginal $q$ is the shadow price of these tangible assets, and this price determines their accumulation. Accordingly, standard investment regressions use private fixed investment as a measure of this accumulation. The fact that firms may own other productive assets explains why average $Q$ is a poor proxy for marginal $q$, as the former reflects the value of all assets, while the latter captures the value of fixed capital only.

In a recent paper, Hall (2001) argues that the interpretation of capital should include intangible assets, valued at their own shadow price. That is, Hall suggests that the presence of intangible capital also causes fluctuations in average $Q$ conventionally defined that occur over and above the variations due to fixed capital adjustment costs. By supposing an accumulation process and a demand schedule for intangibles $E$ similar to that of tangible capital $K$, Hall decomposes the value of the firm into the shadow value of both capital stocks:

$$
V=q^{K} K+q^{E} E
$$


Dividing both sides by $K$ shows that the wedge between average and marginal $q$ is simply $q^{E} \frac{E}{K}$. However, because neither the stock of intangibles $E$ nor its shadow price $q^{E}$ are observable in his framework, Hall infers the total shadow value of intangibles by stipulating a series for the shadow value of physical capital $q^{K}$. In other words, viewed from the decomposition equation (1), Hall supposes that average $Q$ is a fair representation of valuation and computes a $q$ series from a number of theoretical assumptions to infer $q^{E} \frac{E}{K}$. In contrast, this paper constructs the omitted variable $q^{\pi}$ directly and analyse this series' contribution to fluctuations in $Q$.

To reiterate this point, consider the following static relationships, which underpin the dynamic stochastic setting which was developed above. Hall considers that inputs, including intangibles, are paid their marginal products, so that

$$
Y=r K+w N+r_{E} E
$$

where $r_{E}$ is the competitive return to intangibles. To simplify further, consider Abel and Blanchard's (1986) definition of dividends, which abstracts from investment: $D \equiv Y-w N=$ $r K+r_{E} E$. The value of the firm is the capitalized value of its future dividends,

$$
r V=D
$$

which implies that

$$
Q=q+\frac{r_{E} E}{r K},
$$

where 'marginal' $q$ is equal to 1 in the long-run. This paper, on the other hand, assumes that firms extract pure profits from downward-sloping demand curves and non-convex cost structures. That is, repeating equation (4):

$$
Y=\pi(r K+w N) .
$$

In this case,

$$
Q=q+\frac{\pi-1}{\pi} \frac{Y}{r K},
$$


which is to be compared to (10) ${ }^{7}$.

Both frameworks attempt to document the disconnect between average and marginal $q$ by formalising a missing variable bias. Yet, despite the overlap in the interpretation of intangibles and monopoly rents, the methodology involved is substantially different. In the first case, the bias arises from the mismeasurement of capital and the oversight of intangibles. In the second, it arises because inputs are not paid their marginal products. A crucial difference is that, as the next section shows, the latter bias is computable independently of either $q$ or $Q$, so that Hall's (strong) requirement that current levels of $Q$ be a fair representation of valuation is not necessary.

Another essential difference lies in the long-run properties of the two models. Referring to equations (10) and (11), it seems uncontroversial that marginal q should be stationary (it is a cost derivative, so if it was integrated of order 1 , total cost would be $\mathrm{I}(2)$, an order higher than output). Thus average $\mathrm{Q}$ is $\mathrm{I}(0)$ if and only if the ratio on the RHS of either equation is $\mathrm{I}(0)$. Equation(10) suggests that average $Q$ is stationary only if tangibles and intangibles are cointegrated. Equation (11), on the other hand, shows that average $Q$ mean reverts to 1 only if entry precludes long-run monopoly rents. The underlying mean-reversion mechanisms need not be the same.

The intangibles and rents biases can be combined in a single model. Re-writing equation (4) so that it includes the payments to intangibles,

$$
Y=\pi\left(r K+w N+R_{E} E\right)
$$

7 Equation (11) points to the bias, discussed later in Section 4, that affects the $q$ series derived in Abel and Blanchard (1986). Their operational measure of the average profit of capital is

$$
\frac{D}{K}=r+\frac{\pi-1}{\pi} \frac{Y}{K}=\tilde{r}
$$

while the marginal product of capital is $r$. This implies that their measure of marginal $q$, which is the capitalized value of these future average profit rates, is actually a measure of average $q$ :

$$
\frac{V}{K}=\frac{V}{D} \frac{D}{K}=\frac{\tilde{r}}{r}
$$

If $\pi \neq 1$, the induced bias in this $q$ series may have misled these authors in the analysis of the correlation between $q$ and investment (or $K$, in this simple example). 
produces the following expression for average $Q$ :

$$
Q=q+\frac{r_{E} E}{r K}+\frac{\pi-1}{\pi} \frac{Y}{r K}
$$

This equation points to the relative merits of the two frameworks. Hall assumes perfect competition and constant returns to scale (i.e. $\pi=1$ ), and attempts to compute $E$ by postulating $r_{E}$ and a process for marginal $q$ and subtracting the latter from average $Q$. Beyond the inherent arbitrariness of actually postulating the unobservable $q$ process (more specifically the value of the stable root of his system), his implied measure of $r_{E} E$ could be grossly inaccurate if $\pi$ actually differs from 1 . On the other hand, this paper is concerned with the empirical connection between the measurable value of rents and average $Q$. However, subtracting the former from the latter does not produce the right measure of marginal $q$ for the purpose of analysing the behavior of fixed investment, because of the second term in (12), the 'noise' that stems from the presence of intangibles.

Clearly, this paper's framework could include Hall's methodology for constructing $q^{*}$ in (12). Given that the third term on the right-hand side can be computed independently of the other two, as the next section shows, this would enable us to decompose valuation into three terms: the productivity of physical capital, of intangible capital, and monopoly rents. For conciseness, however, this paper argues that the third term is of valuable interest in itself. The next section focuses exclusively on rents and their relation to average $Q$. Thus, in what follows, intangibles are ignored and a full empirical analysis of (12) is left for future research.

\section{Estimating co-movements of $Q$ and monopoly rents}

With reference to equation 1, this section analyses the extent to which $q, q^{\pi}$ and the part of $q^{\pi}$ which is orthogonal to $q$ contribute to movements in $Q$. This decomposition is attempted in levels. Since the steady-state value of $q^{\pi}$ is zero from the assumption of free entry, $q^{\pi}$ can be replaced by its percentage deviation from one as defined in subsection 2.2 , so that

$$
Q=q+\hat{q}^{\pi}
$$

Since $\hat{q}^{\pi}$ is an expected present value variable, one must determine the time-series prop- 
erties of the information set over which these expectations are formed. Subsection 3.2 looks at a simple univariate process, while subsection 3.3 considers a VAR process. Interest in the latter is threefold: assessing the impact of the choice of information set, gauging the importance of second-order terms in the approximation performed for equation (9), and computing a version of Abel and Blanchard's (1986) q series for Section 4. Subsection 3.4 comments on the VAR estimation. Subsection 3.5 present the central results concerning the comovements of average $Q$ and the various computed series of $\hat{q}^{\pi}$.

\subsection{Rents and the labor share}

To compute the first-order approximation for the $\hat{q}^{\pi}$ process, consider the optimality condition along the labor margin, which is also the operational definition of the markup $\mu$ as the ratio of price (here, normalized to 1) to marginal cost (the ratio of wage to marginal product):

$$
F_{N}\left(K_{t}, N_{t}\right)=\mu_{t} w_{t}
$$

Multiply and divide by the labor input, use the assumption that the production function $F$ is linear homogeneous in its inputs and the definition of the returns to scale index $\eta=F y^{-1}$ to obtain

$$
\begin{aligned}
\alpha & \equiv \frac{N_{t} F_{N}\left(K_{t}, N_{t}\right)}{F\left(K_{t}, N_{t}\right)}=\mu_{t} \frac{Y_{t}}{F\left(K_{t}, N_{t}\right)} \frac{w_{t} N_{t}}{Y_{t}} \\
\alpha & =\frac{\mu_{t}}{\eta_{t}} s_{t} \equiv \pi_{t} s_{t} .
\end{aligned}
$$

The standard assumption in much of the macroeconomic literature about the labor elasticity of output is that it is equal to the labor share. Yet as equation (13) points out, this is only true if there are no pure profits $(\pi=1)$. Inversely, if this elasticity is constant-as in the Cobb-Douglas framework used in this paper - movements of the labor share must be matched by offsetting movements in the pure profit share. This implies the two fundamental points of the paper. Although individual series for $\mu_{t}$ and $\eta_{t}$ are of interest in themselves and have spawned a large literature, only their ratio matters for valuation purposes, and this ratio is simply captured by movements in the observable labor share. Second, tautologically within the class of Cobb-Douglas production functions, the labor share does not depend on assump- 
tions about the behavior of the capital margin. That is, no assumptions are necessary about capital adjustment costs to compute the pure profit share, since to a first approximation, the latter can be computed from the labor margin only.

\subsection{Simple univariate process}

The data used throughout the paper is described in Appendix 6.5. The procedures below follow Abel and Blanchard (1986) in that all variables that enter the computation of $\hat{q}^{\pi}$ are exponentially detrended before any estimation is done. Therefore, this paper has nothing to say about trend movements. The choice of modelling trends as deterministic is controversial, but I simply follow the standard business cycle literature convention. Lafourcade (2003b) examines similar correlations within a stochastic trend framework.

Figure 2 plots the logarithm of the corporate sector labor share over the past 50 years. This series exhibits relatively pronounced movements. As figure 3 shows, the quarterly labor share seems to follow an $\operatorname{AR}(1)$ process with an autocorrelation coefficient $\hat{\phi} \simeq 0.925$. $\mathrm{Al}$ though not reported here, ADF and Phillips-Perron tests confirm that the labor share (before detrending) is a mean-reverting series over the period 1952Q1 to 2001Q3 (which is the sample this paper will focus on, mainly because the Federal Reserve's data for capital is available only in quarterly format from 1952 onwards). Thus at first pass, the profit rate's dynamic behavior lies just short of the region of interest shown in figure 1 where the magnifying effect is reinforced. Contrary to the approach Rotemberg and Woodford (1999) take, where these authors limit a similar analysis to the subperiod 1973Q1 to 1993Q1 in view of the possible structural breaks at the onset of the 1970s and 1990s, this paper will assume that the process for the labor share is stationary and invariant over the entire post-war period. ${ }^{8}$

From (9), the $\hat{q}^{\pi}$ series is defined by

$$
\hat{q}_{t}^{\pi}=-\frac{r R}{(1-\alpha) \rho} \frac{\rho \hat{\phi}}{1-\rho \hat{\phi}} \hat{s}_{t} \equiv f(\hat{\phi}) \hat{s}_{t} .
$$

Note that $\hat{q}_{t}^{\pi}$ is a discounted sum of variables, starting at time $t+1$, whose expectations are

\footnotetext{
${ }^{8}$ It is well-known that estimates of $\mathrm{AR}(1)$ coefficients are biased downward in small samples, but the bias runs the other way in the presence of structural breaks. Investigating whether these two biases offset each other in the unit-root test of the labor share is, however, beyond the scope of this paper.
} 


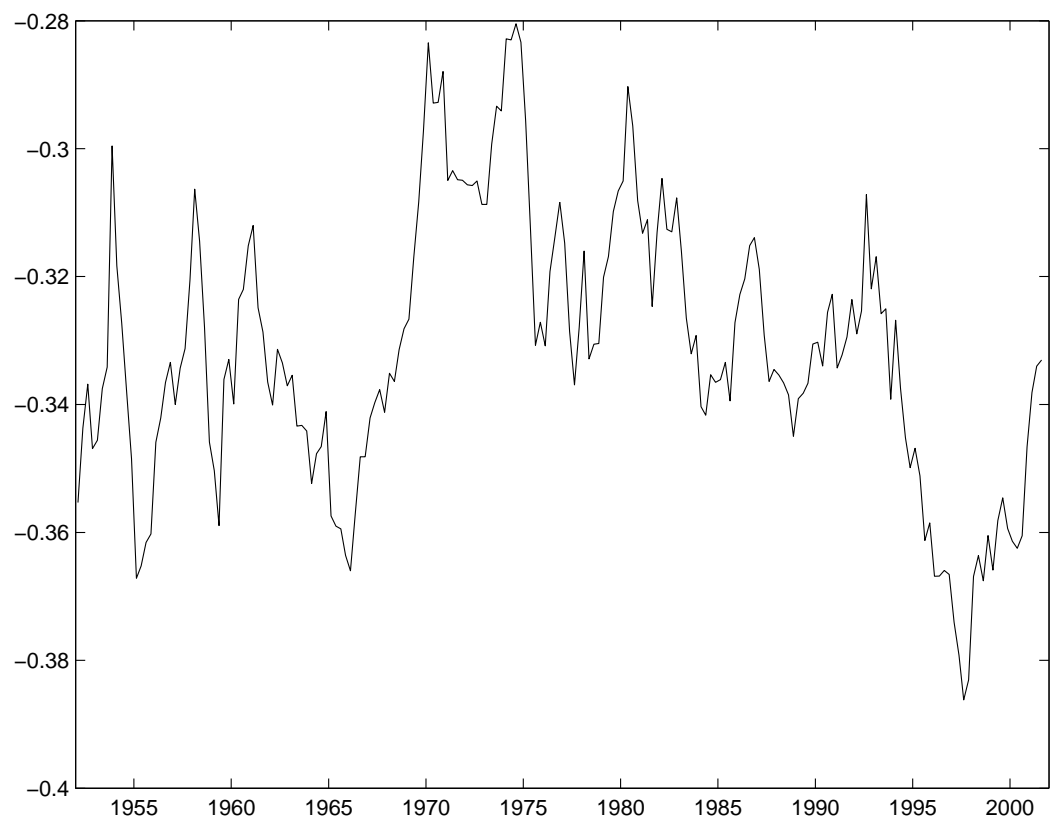

Figure 2: Logarithm of the corporate labor share

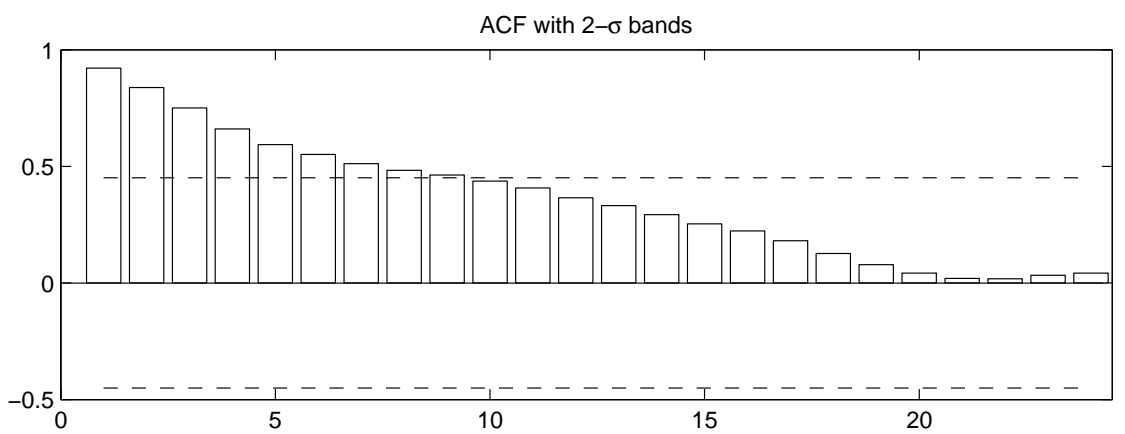

Partial ACF with $2-\sigma$ bands

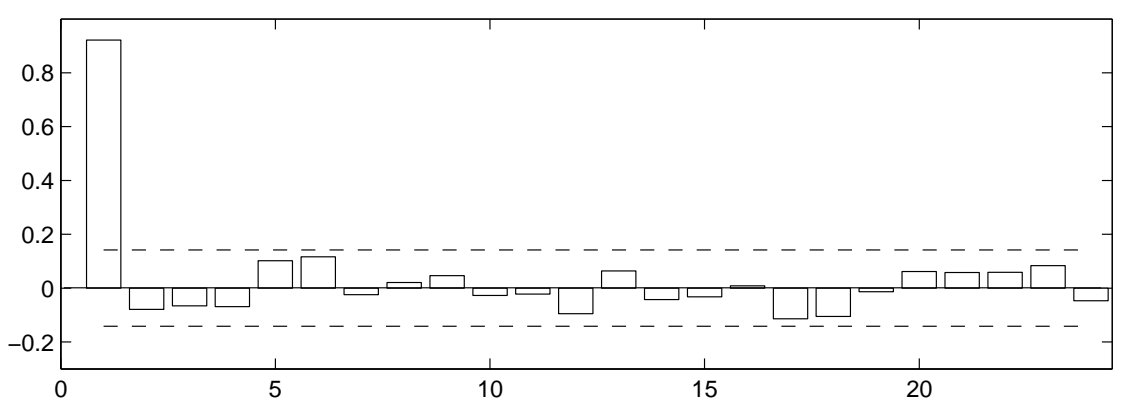

Figure 3: Quarterly time-series properties of the labor share 


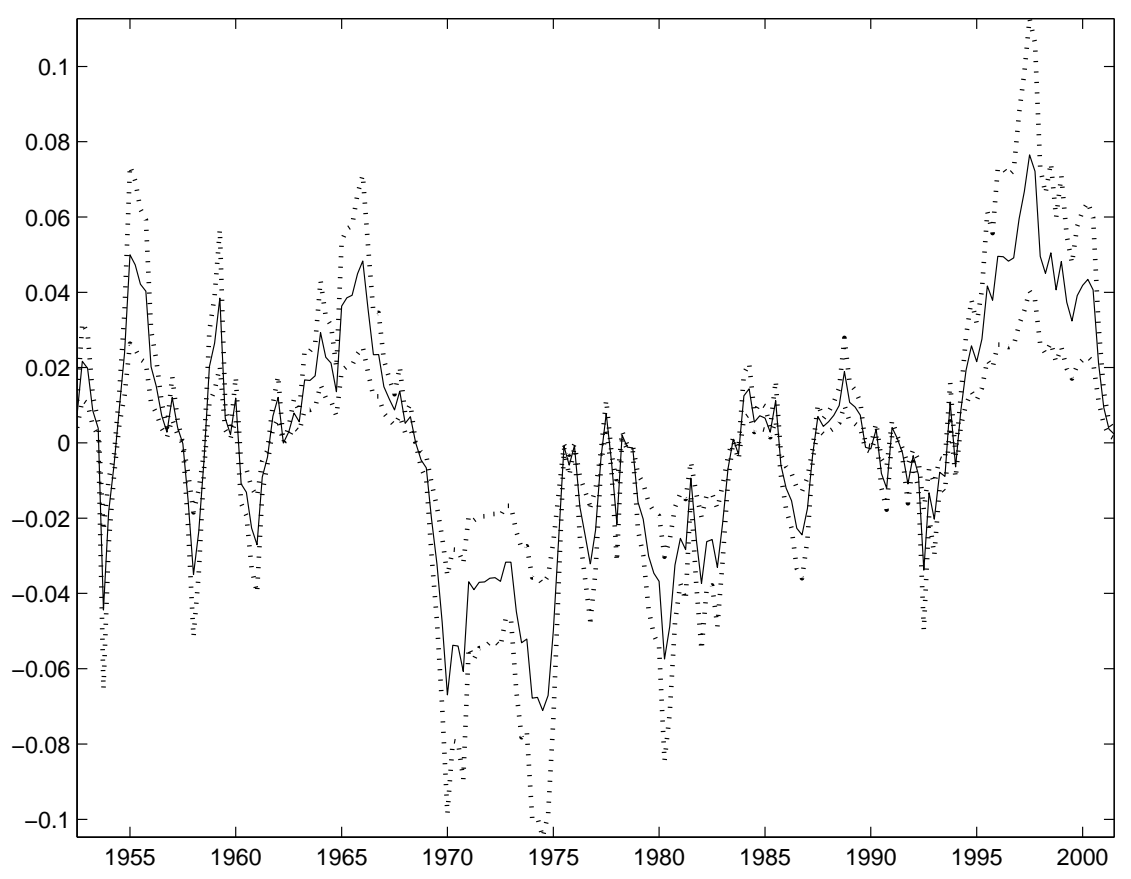

Figure 4: Estimated PDV of pure profits and root mean squared error bands

determined with information available at time $t$. Although the labor share $\hat{s}_{t}$ is part of this information set, the parameter $\phi$ is not known with certainty. Appendix 6.3 discusses the computation of parameter uncertainty bands around point estimates of a univariate $\operatorname{AR}(1)$ process such as $\hat{q}_{t}^{\pi}$, and argues for the use of a second-order version of the delta method.

Figure 4 plots the series with the associated two RMSE bands over the period 1952Q3 to 2001Q3. Observe that the uncertainty band is quite tight, although it increases for large departures from the sample mean, particularly in the early-70s and mid-90s. For the rest of the analysis, this sample path of $\hat{q}^{\pi}$ is assumed to be the notional one. Figure 6 on page 25 plots it against the level version of average $Q$, demeaned over the same period. The assumption that underlies this plot is that $Q$ is a cointegrating vector, hence that it is stationary (an issue dealt with below).

\subsection{Multivariate process and second-order approximation}

This subsection provides two amendments to the computations above. First, it estimates the time-series properties of the labor share in a vector-autoregressive framework, as the VAR may provide more accurate forecasts than univariate filtering by exploiting information from 
other macroeconomic variables. Second, it extends the Taylor expansion that led to equation (9). Indeed, the derivations in subsection 2.2 omitted cross-correlation terms that appear in the form of a discounted sum. Yet, the infinite sum of discounted second-order terms could potentially have first-order effects on $\hat{q}^{\pi}$. Conveniently, these second-order terms will be captured by the same VAR framework.

Including quadratic terms in (9) leads to the amended equation

$$
\hat{q}_{t}^{\pi}=\frac{r R}{(1-\alpha) \rho} \sum_{i=1}^{\infty} \rho^{i} E_{t} \hat{\pi}_{t+i}\left(1+\hat{R}_{t, t+i}+\hat{G}_{t+1, t+i+1}+\hat{m}_{t+i}\right)
$$

where the terms in parentheses are the trend deviations of the compound discount factor, the compound (nominal) growth rate of capital and the nominal output-capital ratio, respectively.

To compute the expected cross-correlations, the information set over which expectations are formed needs to be specified. Following the procedure used in Abel and Blanchard (1986) and Rotemberg and Woodford (1996), this set is captured by a VAR which contains the logarithms of the growth rate of real output, the average propensity to consume, detrended hours worked, the nominal output-capital ratio, output price inflation and the labor share. That is, expectations are captured by the first-order companion system:

$$
\begin{aligned}
Z_{t+1} & =A Z_{t}+\varepsilon_{t+1}, \\
Z_{t}^{\prime} & =\left[\begin{array}{lll}
X_{t}^{\prime} & X_{t-1}^{\prime} & \ldots
\end{array}\right], \quad \varepsilon_{t} \hookrightarrow \operatorname{IID}(0, \Omega), \\
X_{t}^{\prime} & =\left[\begin{array}{llllll}
\Delta \hat{y}_{t} & \hat{c}_{t}-\hat{y}_{t} & \hat{n}_{t} & \hat{m}_{t} & \Delta \hat{p}_{t} & \hat{s}_{t}
\end{array}\right] .
\end{aligned}
$$

The VAR is a crude way of capturing sample correlations of the constituents of the $\hat{Q}$ series, and can be seen as the reduced form of a structural model whose inner workings however are not the purpose of this paper. The dynamics of the system composed of the first three elements have been extensively studied by Rotemberg and Woodford. The last three elements are included to compute the expectations of pure profits. $Z$ is similar to the information vector used by Abel and Blanchard, but differs in the choice of measure for the discount factor. Indeed, they use a weighted average of equity and debt yields, whereas this 
paper uses the consumption-based pricing kernel ${ }^{9}$. This choice bears heavily on results in the next section, and will be discussed in time. Because average $Q$ is computed as a ratio of two nominal variables, $Z$ should capture information about price dynamics, hence the inclusion of inflation. Finally, notice that deviations of the output-capital ratio are defined for end-of-period capital: $\hat{m}_{t}=\left(\hat{p}_{t}+\hat{y}_{t}\right)-\left(\hat{p}_{t}^{k}+\hat{k}_{t+1}\right)$.

All four terms on the right-hand side of equation (15) can be expressed as functions of the $Z$ vector alone.

First, note that the growth rate of capital and the capital-output ratio can be conveniently combined:

$$
\begin{aligned}
\hat{G}_{t+1, t+i+1}+\hat{m}_{t+i} & =\left(\hat{y}_{t+i}-\hat{y}_{t}\right)+\left(\hat{p}_{t+i}-\hat{p}_{t}\right)+\hat{m}_{t} \\
& =\left(e_{1}+e_{5}\right) \sum_{j=1}^{i} Z_{t+j}+e_{4} Z_{t}
\end{aligned}
$$

where $e_{i}$ is the $1 \times 6 n$ vector which selects the $i^{t h}$ element of $Z$, and $n$ is the number of lags in the VAR specification.

Second, consider the consumption-based stochastic discount factor $R$. General equilibrium models have long failed to account jointly for the equity-premium and risk-free rate puzzles (see Kocherlakota (1996) for example). One relatively successful solution to this problem involves generalising the representative consumer's utility function, and more specifically, introducing habit formation in consumption. Although it is generally accepted that Campbell and Cochrane's (1999) additive approach provides a better resolution of the puzzles, this paper adopts Abel's (1998) 'catching up with the Joneses' formulation of one-lagged multiplicative external habits. The reason is purely for convenience, since the former authors' specification requires modelling an extra variable, the consumption-surplus ratio, whereas

\footnotetext{
${ }^{9}$ Abel and Blanchard construct their marginal $q$ measure from the VAR properties of an estimated information set over which the forecasts of the profit function are computed. Bond and Cummins (2001) attack the issue from a different angle, by building a similar series with actual real-time profit forecasts of financial analysts. They confirm the finding that the weak relationship between investment and observed average $q$ can be attributed to the weak relationship between valuation and expectations of future profits. That is, a constructed measure of the right-hand side of equation (1) is more successful than observed average $q$ in explaining investment fluctuations. Although Bond and Cummins' results are derived from a dataset with arguably more potent explanatory content than the aggregate data that Abel and Blanchard use, this paper will remain within the VAR framework developed by the latter, and will attempt to quantify the added value of accounting for the presence of monopoly rents.
} 
Abel's formulation is entirely captured by the $Z$ vector described above.

Indeed, in the latter case, the discount factor is computed as

$$
R_{t, t+i}=\beta^{i}\left[\frac{C_{t+i}}{C_{t}}\right]^{-\gamma}\left[\frac{C_{t+i-1}}{C_{t-1}}\right]^{\kappa(\gamma-1)}
$$

where $\gamma$ is the coefficient of risk aversion and $\kappa$ governs the degree of time-non-separability. ${ }^{10}$ The leverage of this formulation is that a high $\kappa$ compensates a high $\gamma$, so that the coefficient of risk aversion can be raised to solve the equity premium puzzle without compounding the riskless rate puzzle ${ }^{11}$. For the purpose of the paper, time-nonseparability increases the volatility of marginal utility for a given degree of smoothness of consumption, a feature which could boost the cross-correlation terms involved in equation (15).

Conveniently, this specification leads to the deviation form

$$
\begin{aligned}
\hat{R}_{t, t+i} & =-\gamma\left(\hat{c}_{t+i}-\hat{c}_{t}\right)+\kappa(\gamma-1)\left(\hat{c}_{\bar{t}+i-1}-\hat{c}_{t-1}\right) \\
& =e_{R 1}\left(Z_{t}-Z_{t+i}\right)-e_{R 2} \sum_{j=1}^{i} Z_{t+j}
\end{aligned}
$$

where $e_{R 1} \equiv \gamma e_{2}-\kappa(\gamma-1) e_{8}$ and $e_{R 2} \equiv \gamma e_{1}+\kappa(\gamma-1) e_{7}$. Replacing in (15), one obtains the measure of $\hat{q}^{\pi}$ consistent with the information vector $Z$ :

$$
\frac{(1-\alpha) \rho}{r R} \hat{q}_{t}^{\pi}=-e_{6}\left[T_{1 t}+T_{2 t}\left(e_{R 1}+e_{4}\right)^{\prime}-T_{3 t}\left(e_{R 1}\right)^{\prime}+T_{4 t}^{\prime}\left(e_{1}+e_{5}-e_{R 2}\right)^{\prime}\right]
$$

where the $T_{i}$ terms are derived in Appendix 6.2. The term $T_{1}$ is the VAR-equivalent of 'univariate' $\hat{q}^{\pi}$ which was constructed in the previous subsection, while $T_{2}, T_{3}$, and $T_{4}$ are the quadratic correction terms.

\subsection{VAR estimation}

A detailed consideration of the point estimates of the VAR is of limited interest, and the results are reported in Appendix 6.1. Table 3 reports the VAR results obtained over the period

\footnotetext{
${ }^{10}$ Note that the condition $\kappa(\gamma-1)>0$ is required for habit formation to affect the discount factor.

${ }^{11}$ At the same time, however, a high $\kappa$ produces counterfactual volatility in the expected real interest rate (see Campbell et al. (1997)).
} 
1952Q1 to 2001Q3. The autoregression includes only two lags, so as to avoid overfitting. This point notwithstanding, the choice of two lags is consistent with the Akaike information criterion.

As should be expected when looking at raw data over the past fifty years, the VAR estimation exhibit symptoms of econometric misspecification. Indeed, standard augmented Dickey-Fuller tests applied to the output-capital ratio $m$ (not reported here) do not reject the null hypothesis of non-stationarity. In the current framework, this translates into some of the eigenvalues of the estimated matrix $\hat{A}$ lying close to the unit circle, a result that bears on the elements of the long-run multiplier matrix $\hat{B}=(I-\rho \hat{A})^{-1}$ that feeds in turn into the $T_{i}$ terms. The amplitude of these eigenvalues is reported in Table 4. However, from a theoretical point of view, and with Kaldor's stylized fact in mind of a roughly constant capital-ouput ratio, it seems reasonable to assume that the ratio is bounded in the long-run. In fact, the failure of the tests is most probably due to the size of the sample. Regardless, insofar as the main purpose of the VAR is to extract in-sample forecasts, the issue of non-stationarity is minor and the sample path of $\hat{q}^{\pi}$ seems consistent with stable roots.

Nevertheless, if one assumes on the basis of the unit root tests that the capital-output ratio is $I(1)$, the least square regressions of the other variables are unbalanced because they capture correlations between series of different orders. To account for this problem, the VAR is also estimated with the restriction that the parameters of the output-capital ratio are zero at all lags for all equations except its own. The results are reported in the bottom panel of Table 3. This is done to restore balance between regressors and regressands. Although the matrix $\tilde{A}$ of coefficients derived under these restrictions may coincidentally be the same as the 'unrestricted' matrix $\hat{A}$, the computation of the matrix $B$ of long-run multipliers could compound marginal differences up to a substantial size. The results that follow are therefore presented under both sets of coefficients $\hat{A}$ and $\tilde{A}$. Performing correlation analysis along both information sets is a useful gauge of how sensitive the results are to the point estimates.

Another point worth emphasising is that the assumption that the estimated $\operatorname{VAR}(2)$ is the correct representation of how expectations are formed forces the labor share to follow a second-order process that is quite different from the univariate one derived in subsection 3.2.

Lastly, because the matrix $\hat{A}$ is stochastic, standard error bands are computed for the 


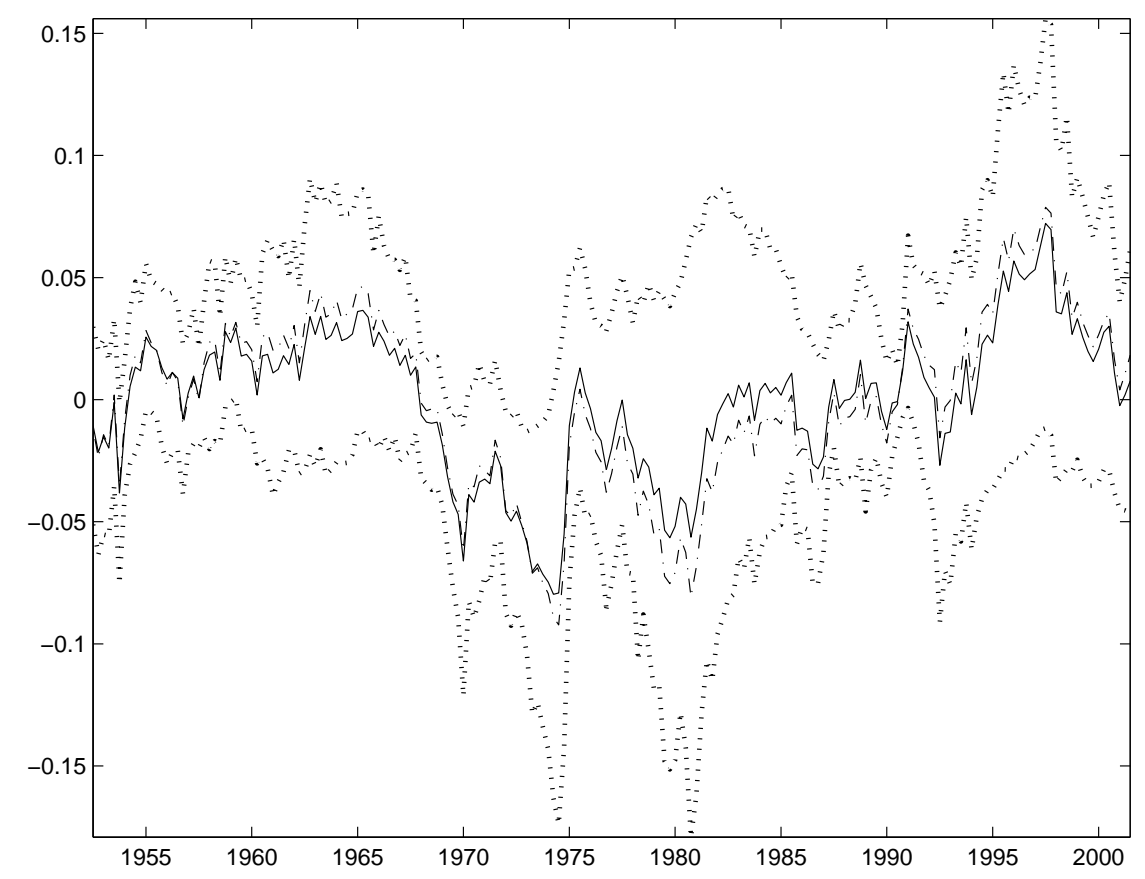

Figure 5: VAR-estimated PDV of pure profits (unrestricted - solid, restricted - dashed) with standard error bands

VAR-based constructed measure of $\hat{q}^{\pi}$. This is done for both information sets. Appendix 6.4 derives the necessary computations. Note that the bands are wider than in the univariate case. One reason is that, as Appendices 6.3 and 6.4 discuss, the univariate error bands are corrected for small-sample bias and a second-order approximation of the delta method, whereas the multivariate error bands are not. Another reason is that the bands weigh more heavily the more poorly estimated parameters of the other VAR regressors of the labor share (the row of $\hat{A}$ corresponding to $s_{t}$ ). These points notwithstanding, in the light of the stationarity problem discussed above, the error bands are only a relatively weak indicator of the confidence one has in the computed series tracking the notional one. Figure 5 plots $\hat{q}^{\pi}$ under both information sets and the assumption of logarithmic utility, with the standard error bands of the restricted case. The fact that the two series are visually nearly coincidental seems to indicate that the restriction imposed through $\tilde{A}$ is not particularly stringent. This assertion holds for variations in the coefficients of risk aversion and time-non-separability. 


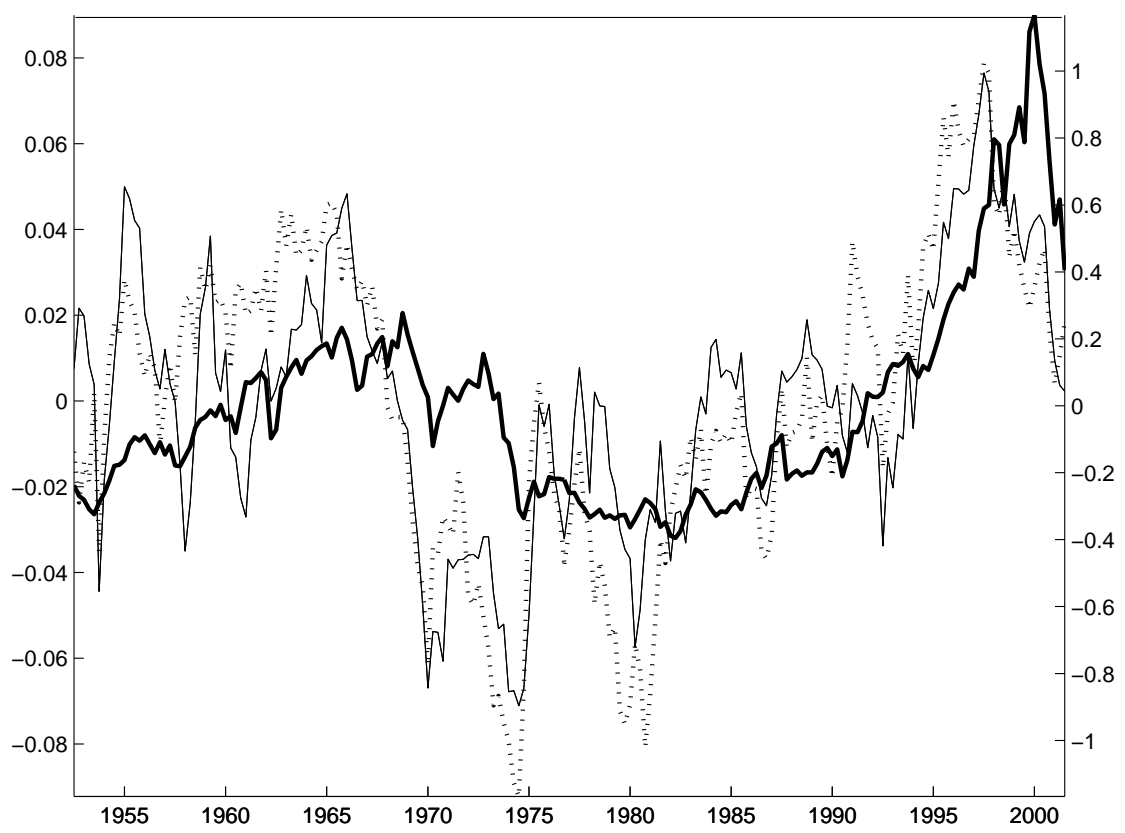

Figure 6: $\hat{Q}$ (thick - right) vs univariate (solid - left) and VAR $\hat{q}^{\pi}$ (dotted - left)

\subsection{Results}

This subsection analyses the correlations and variances between average $Q$ and the measures of $\hat{q}^{\pi}$ computed under different information sets. Comparing $Q$ and $\hat{q}^{\pi}$ is somewhat problematic. Indeed, in the case of linear $\hat{q}^{\pi}$, the labor share is assumed to be $I(0)$ over the sample period, but standard ADF tests do not reject the hypothesis that $Q$ is a unit-root process. More specifically, it is difficult to discriminate between an AR(1) process with high autocorrelation coefficient and a simple random walk. Again, this goes against theory, as it implies that the residually-defined marginal $q$ series (a measure of real marginal cost) is non-stationary. This problem is probably due to the sample size and the notoriously low power of unit-root tests to discriminate between such cases. To avoid tedious analysis of orders of integration, the innovations of both processes are compared instead of the variables themselves. Since $\hat{q}^{\pi}$ is a component of $Q$, its innovation is also a component of the latter's innovation, regardless of what process $Q$ follows. One can therefore analyse the correlation and size properties of these innovations, thus giving an indication of the contribution of $\hat{q}^{\pi}$ to fluctuations in $Q$. Both measures of discounted pure profits are plotted against average $Q$ in figure 6 (the VAR-estimated one having been computed under the assumption of logarithmic 
utility).

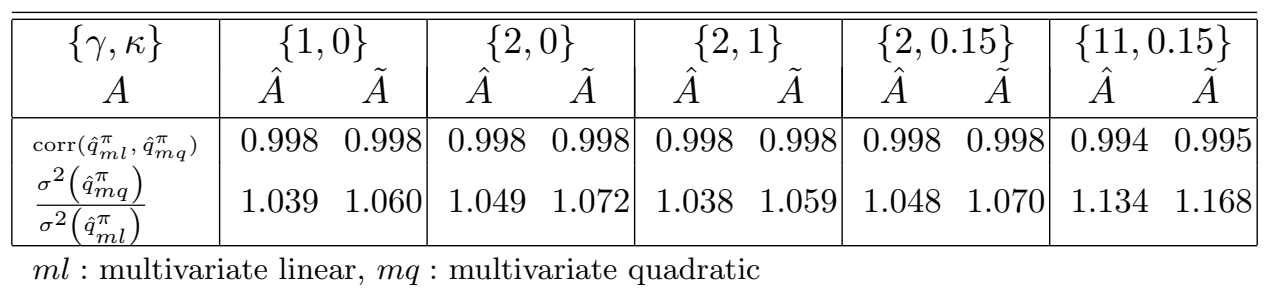

Table 1: Correlation and relative variances of linear vs quadratic $\hat{q}^{\pi}$

In the first instance, table 1 reports results on the usefulness of the quadratic approximation. This approximation depends on a number of features, three of which are the focus here: the coefficient of risk aversion $\gamma$, the habit formation coefficient $\kappa$, and the information set over which the expectations are computed. The parameters $\alpha, R$ and $G$ (and hence $\rho \equiv R G)$ are calibrated as in section 2.2 , so that the discount rate $\beta$ is assumed to 'clear' the habit-formation Euler equation. Hence this is the framework of the first part of figure 1. The table reports the benchmark case of logarithmic utility in the first two columns, and then variations on $\gamma$ and $\kappa$. Notice that the quadratic approximation is for all intents and purposes unnecessary, as the correlation with the linear approximation is almost perfect. Moreover, the former is only very slightly more volatile than the latter. That is, the quadratic correction terms $T_{2}, T_{3}$, and $T_{4}$ have minute effects on the computation of $\hat{q}^{\pi}$. These results are similar in spirit to those developed in Abel and Blanchard (1986). The last two columns report the results for the parameter values chosen by Abel (1998), which involve a higher coefficient of risk aversion than usually accepted. The habit-induced 'kick' to cross-correlation terms that was hoped for does not materialize. In other words, the second-order feature of crosscorrelations, even in present discounted form, dominates this attempt to engineer greater volatility of marginal utility.

Given the practical irrelevance of the quadratic approximation, table 2 focuses on statistics concerning co-movements between average $Q$ and linear measures of $\hat{q}^{\pi}$. The difference between the latter two resides in the estimation of the process for the labor share. In the univariate case, the labor share is $\operatorname{AR}(1)$ whereas it is $\operatorname{ARDL}(2,2)$ in the multivariate case, so that the choice of information set also matters for the latter. Moreover, this table takes the framework of the second part of figure 1, where the subjective discount rate is held 


\begin{tabular}{|ll|lll|lll|}
\hline \hline \multicolumn{4}{c}{ Correlations between $q, \hat{q}^{\pi}$ and $Q=q+\hat{q}^{\pi}$} \\
\hline & \multicolumn{3}{c|}{ univariate } & \multicolumn{3}{c|}{ multivariate linear } \\
\hline & & $\hat{q}_{u}^{\pi}$ & $q_{u}$ & $\hat{q}_{m l}^{\pi}$ & $q_{m l}$ & $\operatorname{corr}\left(\hat{q}_{m l}^{\pi}, \hat{q}_{m q}^{\pi}\right)$ \\
$\gamma=1$ & $A=\hat{A}$ & 0.543 & 0.996 & 0.572 & 0.995 & 0.998 \\
& $A=\hat{A}$ & & & 0.471 & 0.996 & 0.998 \\
$\gamma=2$ & $A=\hat{A}$ & \multirow{2}{*}{0.996} & 0.566 & 0.995 & 0.998 \\
& $A=\hat{A}$ & & 0.472 & 0.996 & 0.998 \\
\hline
\end{tabular}

Fractions of the innovation variance of $Q$ accounted by $\hat{q}^{\pi}$ and $q$

\begin{tabular}{|cc|ccc|ccc|}
\hline \multicolumn{3}{|c}{} & \multicolumn{3}{c}{ univariate } & \multicolumn{3}{c|}{ multivariate linear } \\
\hline & & $\frac{\sigma^{2}\left(\hat{q}_{u}^{\pi}\right)}{\sigma^{2}(Q)}$ & $\frac{\sigma^{2}\left(q_{u}\right)}{\sigma^{2}(Q)}$ & $\frac{\sigma^{2}\left(\hat{q}_{u}^{\pi} \mid q_{u}\right)}{\sigma^{2}(Q)}$ & $\frac{\sigma^{2}\left(\hat{q}_{m l}^{\pi}\right)}{\sigma^{2}(Q)}$ & $\frac{\sigma^{2}\left(q_{m l}\right)}{\sigma^{2}(Q)}$ & $\frac{\sigma^{2}\left(\hat{q}_{m l}^{\pi} \mid q_{m l}\right)}{\sigma^{2}(Q)}$ \\
$\gamma=1$ & $A=\hat{A}$ & 0.032 & 0.980 & 0.025 & 0.055 & 1.066 & 0.042 \\
& $A=\hat{A}$ & & & & 0.045 & 1.055 & 0.038 \\
$\gamma=2$ & $A=\hat{A}$ & 0.036 & 0.980 & 0.027 & 0.057 & 1.068 & 0.044 \\
& $A=\tilde{A}$ & & & & 0.048 & 1.059 & 0.041 \\
\hline
\end{tabular}

Table 2: Correlations and variances

fixed and the rate of return 'clears' the Euler equation. For comparative purposes, the table reports only the benchmark logarithmic case and the case with $\gamma=2, \kappa=0$, as the statistics were observed to be only mildly sensitive to the choice of these parameters. Indeed, again, habit-formation seems to carry little sway in this framework.

Consider first the univariate case. The two series $\hat{q}^{\pi}$ and $Q$ display a correlation coefficient of 0.543 , which is in fact (minus) the correlation between $Q$ and the labor share, since $\hat{q}^{\pi}$ is simply a multiple of the latter. However, the magnitude of the divergence of scale between the two series is the salient feature of the innovation variances. Indeed, the variance of the innovation in $\hat{q}^{\pi}$ represents little more than $3 \%$ of the variance of $Q$ 's innovation (a fact quite apparent from Figure 6, although the latter displays the scale for the series themselves, not their innovations). By implication, the ratio of innovation variances of the marginal $q$ series to that of average $Q$ is close to 1 .

The table also reports the variance of the innovation component of $\hat{q}^{\pi}$ that is orthogonal to marginal $q$. This statistic is equal to the innovation variance of $\hat{q}^{\pi}$ time $1-\rho^{2}$, where $\rho$ is the correlation coefficient between $\hat{q}^{\pi}$ and marginal $q$. It is quite clear that this orthogonal component explains very little of the volatility of average $Q$. Thus, and this is the central result of the paper, even though $\hat{q}^{\pi}$ is positively correlated with average $Q$, it induces movements 
in the latter that are much smaller than the actual ones. Given that $\hat{\phi}$, the autocorrelation coefficient for the labor share, is an estimated parameter, overturning this result would seem to require implausible assumptions about the subjective discount rate, the depreciation rate and the growth rate, as discussed in subsection 2.2. Hence this first estimate of variations in the measure of monopoly rents has only very modest effects on one's account of the sources of fluctuations in average $Q$, and leaves much to be explained. Admittedly, this void is captured by adjustment costs and the volatility of investment, and/or the presence of intangibles, both of which enter the composition of the residual series $q$.

Consider now the VAR-based measure of discounted pure profits with average $Q$. The fact that this linear construction forces the labor share to follow a second-order autoregressive distributed lag process bears heavily on the correlation of the $\hat{q}^{\pi}$ series with $\hat{Q}$. This correlation is not dependent on assumptions about the coefficient of risk aversion, but is sensitive to the choice of information set. In the unrestricted case, the correlation with average $Q$ is higher than in the univariate case, but this conclusion is reversed with the restricted information set. The same sensitivity applies to the ratios of variances, which are higher than in the univariate case, but still rather small at around 5\%. In fact, the innovation variance of implied marginal $q$ is greater than that of average $Q$, which suggests that innovations in $\hat{q}^{\pi}$ which are negatively correlated with $q$ counterbalance the effects of short-run movements of $q$ on $Q$. In conclusion, on the whole, the VAR-based measure of discounted pure profits provides only little more empirical substance than the univariate measure to the idea that valuation could be strongly dependent on the behavior of rents.

\section{Abel and Blanchard (1986) revisited}

Section 3 concentrated on the relationship between average $Q$ and rents. Yet the results obtained therein do not necessarily extend in an obvious way to the relationship between investment and the rent-free measure of $q$, because observed average $Q$ appears disconnected from the constructed series of discounted future profits (as was discussed in Section 1 and footnote 9).

This section therefore attempts to re-assess Abel and Blanchard's analysis of this relationship in the light of the 'noise' that arises from the presence of pure profits. It also adopts 
their definition of dividends as gross of investment costs, despite the criticisms raised by Bond and Cummins (2001). Furthermore, it does not address Hall's (2001) issue about including intangibles in the computational process, and is thus subject to the caveat developed in subsection 2.3 .

Abel and Blanchard's measure (in levels) of marginal $q$ is

$$
\begin{aligned}
q_{t}^{A B} & =E_{t} \sum_{i=1}^{\infty} R_{t, t+i} \frac{Y_{t+i}-w_{t+i} N_{t+i}}{K_{t+1}} \\
& =E_{t} \sum_{i=1}^{\infty} R_{t, t+i} G_{t+1, t+i+1}\left(1-s_{t+i}\right) m_{t+i}
\end{aligned}
$$

This can be decomposed into the two revenue flows as in subsection 2.2 :

$$
\begin{aligned}
q_{t}^{A B} & =E_{t} \sum_{i=1}^{\infty} R_{t, t+i} G_{t+1, t+i+1}\left(1-\frac{1}{\pi_{t+i}}\right) m_{t+i}+E_{t} \sum_{i=1}^{\infty} R_{t, t+i} G_{t+1, t+i} r_{t+i} \\
& =q_{t}^{\pi}+E_{t} \sum_{i=1}^{\infty} R_{t, t+i} G_{t+1, t+i} r_{t+i}
\end{aligned}
$$

Admittedly, the expression the authors are interested in is the second term on the right-hand side. But their measure of $q$ is polluted by movements in the pure profit share. Using the optimality conditions (7) and (13), the correct measure should be

$$
q_{t}=E_{t} \frac{1-\alpha}{\alpha} \sum_{i=1}^{\infty} R_{t, t+i} G_{t+1, t+i+1} s_{t+i} m_{t+i}
$$

which is identical to (16) only in the case that $s_{t}=\alpha$, or equivalently $\pi_{t}=1$.

Consider the trend deviation version of equation (16):

$$
\begin{aligned}
\hat{q}_{t}^{A B} & =E_{t} \frac{1-\rho}{\rho} \sum_{i=1}^{\infty} \rho^{i}\left(\hat{m}_{t+i}+\hat{G}_{t+1, t+i+1}-\frac{\alpha}{1-\alpha} \hat{s}_{t+i}+\hat{R}_{t, t+i}\right) \\
& =\left\{e_{R 1}+e_{4}+\left[e_{1}+e_{5}-e_{R 2}-(1-\rho)\left(e_{R 1}+\frac{\alpha}{1-\alpha} e_{6}\right)\right] A B\right\} Z_{t}
\end{aligned}
$$


Compare this expression to equation (17) in deviation form:

$$
\begin{aligned}
\hat{q}_{t} & =E_{t} \frac{1-\rho}{\rho} \sum_{i=1}^{\infty} \rho^{i}\left(\hat{m}_{t+i}+\hat{G}_{t+1, t+i}+\hat{s}_{t+i}+\hat{R}_{t, t+i}\right) \\
& =\left\{e_{R 1}+e_{4}+\left[e_{1}+e_{5}-e_{R 2}-(1-\rho)\left(e_{R 1}-e_{6}\right)\right] A B\right\} Z_{t} .
\end{aligned}
$$

Then the correction that takes into account pure profits is

$$
\hat{q}_{t}^{A B}-\hat{q}_{t}=-e_{6} \frac{1-\rho}{1-\alpha} A B Z_{t}
$$

or equivalently,

$$
\hat{q}_{t}^{\pi}=-e_{6} \frac{r R}{1-\alpha} A B Z_{t}
$$

which is the multivariate counterpart to (14).

The results that follow are not comparable to those obtained by Abel and Blanchard for two reasons. First, their $q$ series is computed over a different information set. Indeed, as was mentioned previously, their series for the discount factor is a market-based measure that incorporates the market's perception of debt and equity returns. This paper uses the consumption-based valuation measure instead, and hence carries all the criticisms and failings reported in the debate over modelling the equity premium in general equilibrium settings. Second, these authors incorporate tax and depreciation allowance effects in their measure, which have been overlooked completely in this framework. As was mentioned in Section 1, these effects are most certainly significant. However, this paper does not mean to develop a full econometric analysis of the relationship between investment and $q$. Rather, it only attempts to point out the relative performance of competing constructed measures of $q$.

In fact, the reason for not running the full gamut of econometric tests of the relationship between $I / K$ and the various measures of $q$ is quite apparent in the next set of figures. Figures 7 and 8 depict $\hat{q}^{A B}$ and $\hat{q}$ with the former's standard-error bands, for two starkly differing choices of $(\gamma, \kappa)$. In both cases - and the pattern is similar for all pairs used in table 1 - the standard-error bands suggest quite clearly that one cannot reject the hypothesis that the two measures of $q$ are the same. Figures 9 and 10 plot both measures of $q$ against the detrended investment-capital ratio. Clearly, the choice of parameters affects the ability of $q$ to 


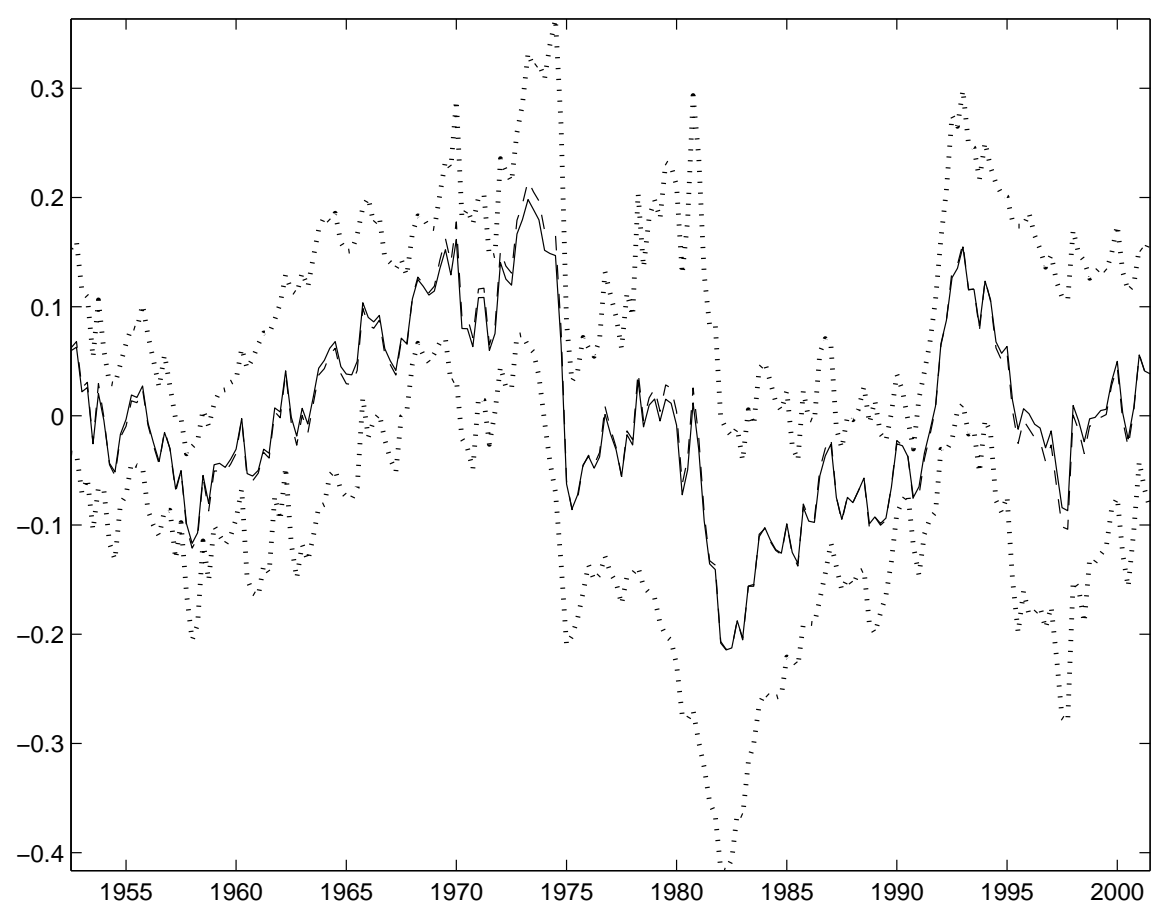

Figure 7: $\hat{q}^{A B}$ (solid) and $\hat{q}$ (dashed) with se-bands. $\gamma=2, \kappa=0, A=\tilde{A}$

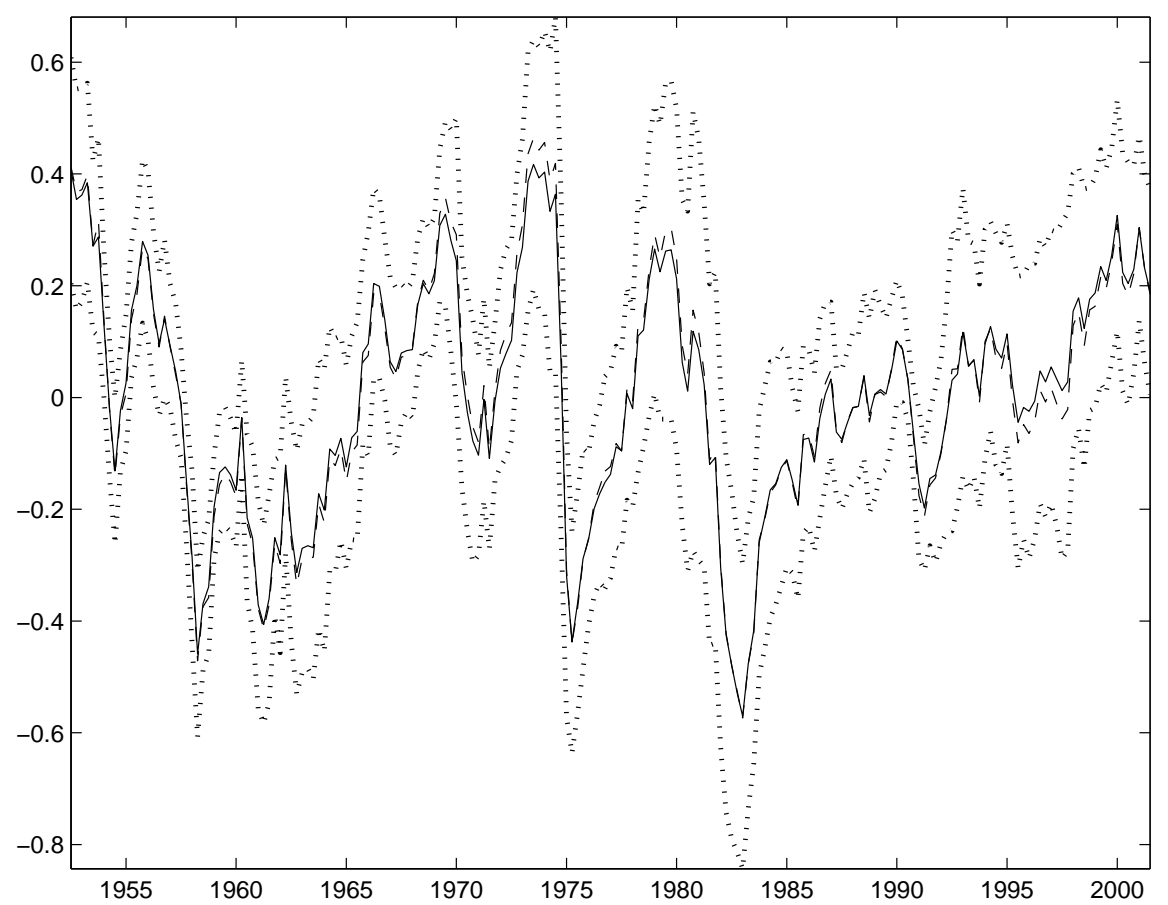

Figure 8: $\hat{q}^{A B}$ (solid) and $\hat{q}$ (dashed) with se-bands. $\gamma=11, \kappa=0.15, A=\tilde{A}$ 


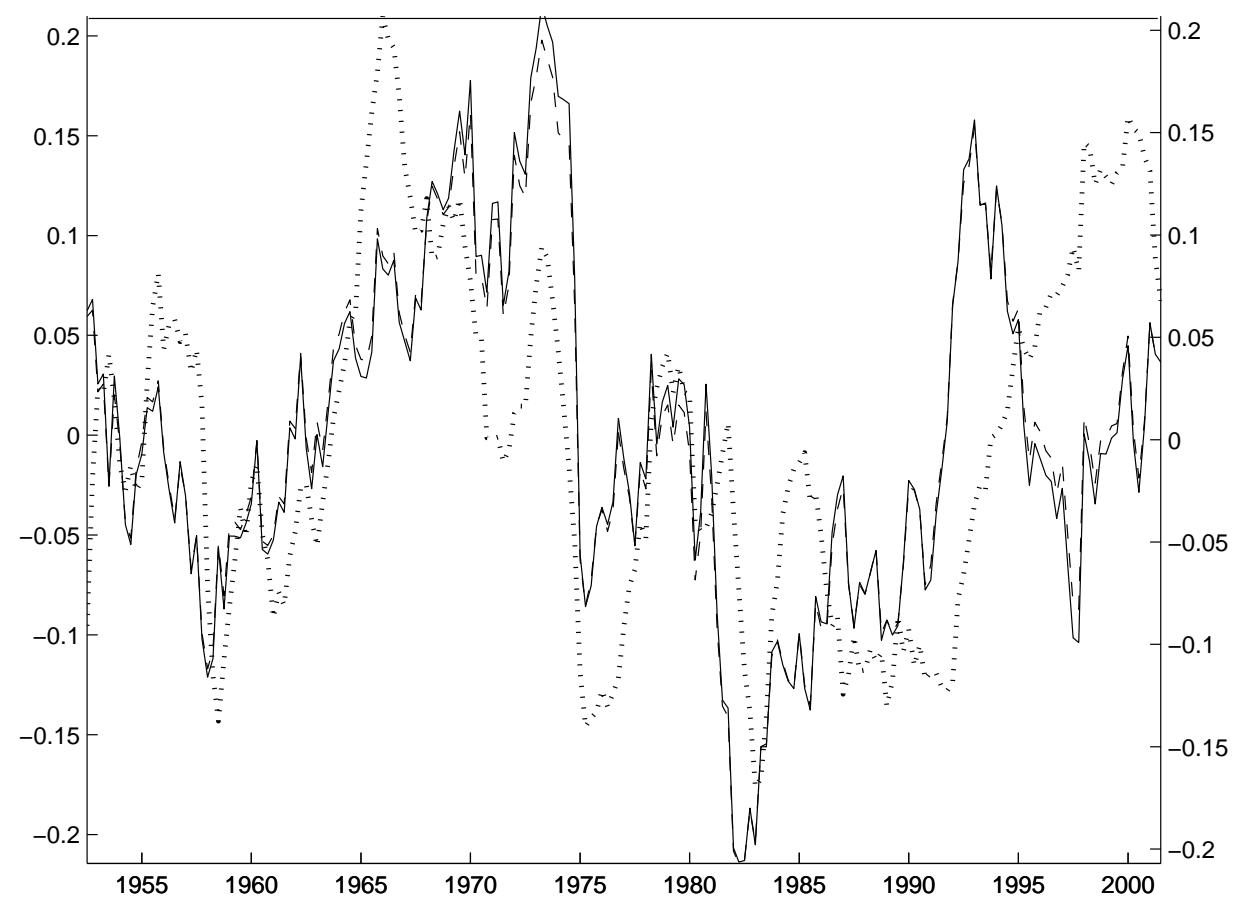

Figure 9: $\hat{q}^{A B}$ (solid) and $\hat{q}$ (dashed) vs $I / K$ (dotted). $\gamma=2, \kappa=0, A=\tilde{A}$

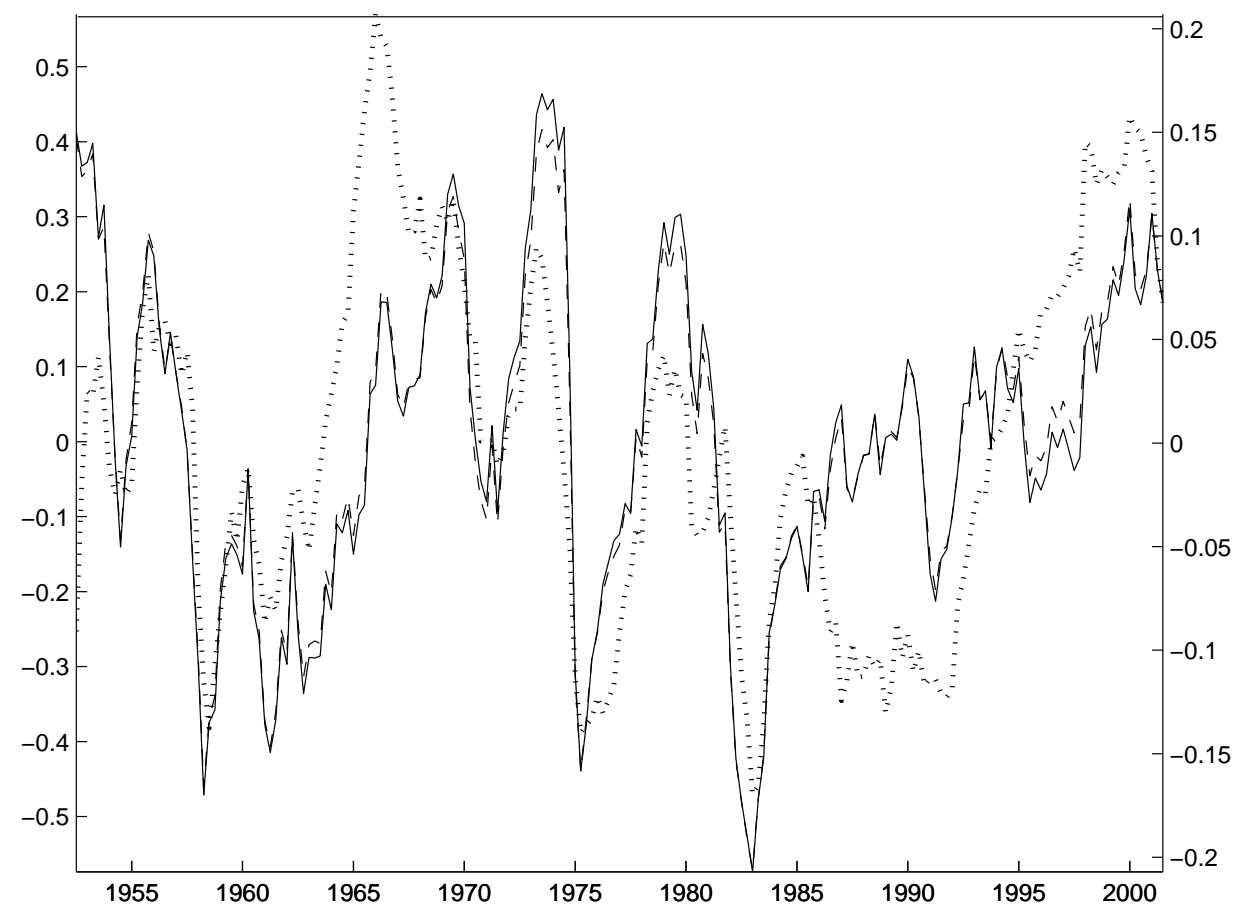

Figure 10: $\hat{q}^{A B}$ (solid) and $\hat{q}$ (dashed) vs $I / K$ (dotted). $\gamma=11, \kappa=0.15, A=\tilde{A}$ 
track investment (note the differences in the two figures for the 1990s). But regardless of this feature, which is the lot of modelling returns with the consumption-based stochastic discount factor, it is again quite evident that for the purpose of explaining investment, the two series are indistinguishable. That is, although movements in rents are significant on their own, they are minute in relation to the discounted value of total profits. The possible corrections to the standard-error bands discussed in Appendix 6.3 are probably not large enough to change these inferences, although this has not been formally tested. In short, the conclusion of this brief section is that although theoretically incorrect, Abel and Blanchard's measure of $q$ is empirically valid enough to warrant econometric analysis of its relation with investment.

\section{Discussion and Conclusion}

The research reported in this paper can be divided into three parts. First, I discussed the theoretical possibility of monopoly power interfering in the standard assumption that average equals marginal $q$. Second, I computed and analysed the properties of a series for the present discounted value of pure profits, and examined its relation to observed movements in average $Q$. Third, I computed two competing measures of $q$, which accounted or not for the existence of these rents, and attempted to assess whether the explanatory power of $q$ for investment behavior was dependent on this assumption. The major finding is that movements in the pure profit share are too small to contribute to any degree to both the size in fluctuations of observed valuation and to the valuation-investment link.

These mixed results can be reassessed along several lines. As was mentioned along the way, attempting to capture the behavior of the discount factor with a consumption-based measure is fraught with difficulties. Yet any success in this field should still have little to say about the present value of monopoly profits, since these rents appear to depend to a first order on the labor share only.

However, this conclusion about first-order dependence could be refined, as it may simply be the figment of the crude econometric specification used for the estimation of expected variables (in equation (15)). The sample paths for rents indicate rather strong sensitivity to the assumptions that lie behind the information set over which expectations are formed. In particular, this paper assumed that trends were deterministic. A follow-up paper (Lafourcade, 
2003b) adopts instead a stochastic trend framework to analyse the cyclical movements in valuation.

Notwithstanding these econometric refinements, the key conclusion of this paper, which is supported by the results in subsections 2.2 and 3.2, is that the labor share does not exhibit the volatility and persistence that are theoretically necessary for rents to mimic the fluctuations of observed average $Q$. However, as Rotemberg and Woodford (1999) argue, there are several reasons why the labor share is only a crude proxy for the pure profit rate. A number of assumptions about production and market structure can be made to correct the labor share in the direction of higher persistence and volatility. This is also investigated in Lafourcade (2003b). 


\section{Appendix}

\subsection{Tables}

Data is described in Appendix 6.5. ${ }^{*}$ indicates significance at $5 \%$ level.

\begin{tabular}{|c|c|c|c|c|c|c|}
\hline $\begin{array}{l}\text { Dependent } \\
\text { variables }\end{array}$ & $\Delta y_{t}$ & $c_{t}-y_{t}$ & $n_{t}$ & $y_{t}-k_{t}$ & $\Delta p_{t}$ & $s_{t}$ \\
\hline & \multicolumn{6}{|c|}{ Unrestricted VAR - sample period 1952:3-2001:3 } \\
\hline \multicolumn{7}{|l|}{ Regressors: } \\
\hline$\Delta y_{t-1}$ & $0.704^{*}$ & $-0.388^{*}$ & $0.486^{*}$ & 0.073 & $0.125^{*}$ & -0.026 \\
\hline$\Delta y_{t-2}$ & -0.011 & 0.019 & -0.026 & -0.039 & -0.026 & $0.119^{*}$ \\
\hline$c_{t-1}-y_{t-1}$ & $1.069^{*}$ & 0.118 & $0.609^{*}$ & $0.895^{*}$ & -0.083 & -0.169 \\
\hline$c_{t-2}-y_{t-2}$ & $-0.763^{*}$ & $0.584^{*}$ & $-0.538^{*}$ & $-0.521^{*}$ & $-0.119^{*}$ & 0.053 \\
\hline$n_{t-1}$ & $0.517^{*}$ & $-0.666^{*}$ & $1.500^{*}$ & $0.496^{*}$ & $0.104^{*}$ & -0.119 \\
\hline$n_{t-2}$ & $-0.471^{*}$ & $0.580^{*}$ & $-0.525^{*}$ & $-0.430^{*}$ & $-0.112^{*}$ & 0.127 \\
\hline$y_{t-1}-k_{t-1}$ & -0.035 & 0.011 & 0.017 & $1.272^{*}$ & $-0.068^{*}$ & 0.052 \\
\hline$y_{t-2}-k_{t-2}$ & 0.028 & -0.001 & -0.018 & $-0.309^{*}$ & $0.065^{*}$ & -0.051 \\
\hline$\Delta p_{t-1}$ & 0.244 & -0.191 & 0.159 & -0.199 & $0.576^{*}$ & 0.202 \\
\hline$\Delta p_{t-2}$ & -0.338 & 88 & -0.113 & -0.569 & $0.172^{*}$ & -0.079 \\
\hline$s_{t-1}$ & $-0.249^{*}$ & 0.2 & -0.127 & $-0.306^{*}$ & $0.073^{*}$ & $1.021^{*}$ \\
\hline$s_{t-2}$ & 0.079 & -0 . & 0.037 & 0.119 & -0.006 & -0.014 \\
\hline Cons & $0.053^{*}$ & 0.039 & $-0.034^{*}$ & -0.055 & $0.024^{*}$ & -0.000 \\
\hline$R^{2}$ & 0.35 & 0.86 & 0.96 & 0.98 & 0.82 & 0.86 \\
\hline & \multicolumn{6}{|c|}{ Restricted VAR - sample period 1952:3-2001:3 } \\
\hline Regressors: & & & & & & \\
\hline $\begin{array}{l}\Delta y_{t-1} \\
\Delta y_{t-2}\end{array}$ & -0.012 & 0.023 & -0.028 & -0.039 & -0.021 & $0.116^{*}$ \\
\hline$c_{t-1}-y_{t-1}$ & $1.076^{*}$ & 0.119 & $0.603^{*}$ & $0.895^{*}$ & $0.102^{*}$ & -0.184 \\
\hline$c_{t-2}-y_{t-2}$ & $-0.776^{*}$ & $0.587^{*}$ & $-0.531^{*}$ & $-0.521^{*}$ & $-0.145^{*}$ & 0.073 \\
\hline$n_{t-1}$ & $0.513^{*}$ & $-0.656^{*}$ & $1.497^{*}$ & $0.496^{*}$ & $0.111^{*}$ & -0.126 \\
\hline$n_{t-2}$ & $-0.474^{*}$ & $0.580^{*}$ & $-0.523^{*}$ & $-0.430^{*}$ & $-0.118^{*}$ & 0.132 \\
\hline$y_{t-1}-k_{t-1}$ & 0.000 & 0.000 & 0.000 & $1.272^{*}$ & 0.000 & 0.000 \\
\hline$y_{t-2}-k_{t-2}$ & 0.000 & 0.000 & 0.000 & $-0.309^{*}$ & 0.000 & 0.000 \\
\hline$\Delta p_{t-1}$ & 0.263 & -0.214 & 0.158 & -0.199 & $0.585^{*}$ & 0.196 \\
\hline$\Delta p_{t-2}$ & -0.281 & 0.212 & -0.120 & -0.569 & $0.220^{*}$ & -0.111 \\
\hline$s_{t-1}$ & $-0.250^{*}$ & $0.269^{*}$ & -0.126 & $-0.305^{*}$ & $0.072^{*}$ & $1.021^{*}$ \\
\hline$s_{t-2}$ & 0.083 & -0.163 & 0.037 & 0.119 & -0.002 & -0.017 \\
\hline Constant & $-0.052^{*}$ & 0.038 & $-0.034^{*}$ & -0.055 & $0.024^{*}$ & -0.000 \\
\hline$R^{2}$ & 0.35 & 0.86 & 0.96 & 0.98 & 0.82 & 0.87 \\
\hline
\end{tabular}

Table 3: Vector Autoregression results

\begin{tabular}{|llllllllll|}
\hline Unrestricted $\hat{A}$ & 0.143 & 0.215 & 0.253 & 0.264 & 0.368 & 0.754 & 0.876 & 0.892 & 0.975 \\
Restricted $\tilde{A}$ & 0.222 & 0.251 & 0.255 & 0.327 & 0.345 & 0.752 & 0.861 & 0.923 & 0.944 \\
\hline
\end{tabular}

Table 4: Amplitude of eigenvalues of $A$ 


\subsection{Quadratic approximation of $\hat{Q}$}

The expression for the VAR-based $\hat{Q}$ series was defined in sub-section 3.3:

$$
\frac{(1-\alpha) \rho}{r R} \hat{Q}_{t}=-e_{6}\left[T_{1 t}+T_{2 t}\left(e_{R 1}+e_{4}\right)^{\prime}-T_{3 t}\left(e_{R 1}\right)^{\prime}+T_{4 t}^{\prime}\left(e_{1}+e_{5}-e_{R 2}\right)^{\prime}\right] .
$$

- $T_{1}$ captures the vector autoregressive form of the discounted sum of future profits rates derived in subsection 2.2 , and can be written as:

$$
T_{1 t} \equiv \sum_{i=1}^{\infty} \rho^{i} E_{t} Z_{t+i}=\sum_{i=1}^{\infty} \rho^{i} A^{i} Z_{t}=\rho A B Z_{t}
$$

where $B \equiv(1-\rho A)^{-1}$ is the matrix of long-run multipliers.

- $T_{2}$ is the element that helps capture the autocovariance between future profit rates and the current information set. Using the fact that the innovation vector $\varepsilon_{t+i}$ is by definition uncorrelated to $Z_{t}$ for $i>0$ yields

$$
\begin{aligned}
T_{2 t} & \equiv \sum_{i=1}^{\infty} \rho^{i} E_{t} Z_{t+i} Z_{t}^{\prime}=\sum_{i=1}^{\infty} \rho^{i}\left(A^{i} Z_{t} Z_{t}^{\prime}+\sum_{j=0}^{i-1} A^{j} E_{t} \varepsilon_{t+i-j} Z_{t}^{\prime}\right) \\
& =\sum_{i=1}^{\infty} \rho^{i} A^{i} Z_{t} Z_{t}^{\prime}=\rho A B Z_{t} Z_{t}^{\prime} .
\end{aligned}
$$

- $T_{3}$ involves the off-diagonal elements of the discounted sum of expected covariance matrices:

$$
\begin{aligned}
T_{3} & \equiv \sum_{i=1}^{\infty} \rho^{i} E_{t} Z_{t+i} Z_{t+i}^{\prime} \\
& =\sum_{i=1}^{\infty} \rho^{i}\left(A^{i} Z_{t} Z_{t}^{\prime} A^{\prime i}+\sum_{j=0}^{i-1} A^{j} E_{t}\left(\varepsilon_{t+i-j} \varepsilon_{t+i-j}^{\prime}\right) A^{\prime j}\right) \\
& =\sum_{i=1}^{\infty} \rho^{i}\left(A^{i} Z_{t} Z_{t}^{\prime} A^{i}+\sum_{j=0}^{i-1} A^{j} \Omega A^{\prime j}\right) .
\end{aligned}
$$


To eliminate the infinite forward sum, notice that

$$
\begin{aligned}
\rho A T_{3 t} A^{\prime} & =\sum_{i=1}^{\infty} \rho^{i+1}\left(A^{i+1} Z_{t} Z_{t}^{\prime} A^{i+1}+\sum_{j=0}^{i-1} A^{j+1} \Omega A^{\prime j+1}\right) \\
& =\sum_{i=2}^{\infty} \rho^{i}\left(A^{i} Z_{t} Z_{t}^{\prime} A^{i}+\sum_{j=0}^{i-1} A^{j} \Omega A^{\prime j}-\Omega\right) \\
& =\sum_{i=1}^{\infty} \rho^{i}\left(A^{i} Z_{t} Z_{t}^{\prime} A^{i}+\sum_{j=0}^{i-1} A^{j} \Omega A^{\prime j}-\Omega\right)-\rho A Z_{t} Z_{t}^{\prime} A^{\prime} \\
& =T_{3 t}-\rho A Z_{t} Z_{t}^{\prime} A^{\prime}-\frac{\rho}{1-\rho} \Omega .
\end{aligned}
$$

This is a discrete Lyapunov equation that can be solved for $T_{3 t}$. It is analogous to the expression derived in Sargent and Ljungqvist (2000) for the autocovariogram of timeseries processes, except that autocovariances are conditional on information at time $t$.

- Finally, $T_{4}$ captures the intermediate autocovariances in the infinite sum:

$$
T_{4 t}=\sum_{i=1}^{\infty} \rho^{i} \sum_{j=1}^{i} E_{t} Z_{t+i} Z_{t+j}^{\prime}=\sum_{i=1}^{\infty} \rho^{i} \sum_{k=0}^{i-1} E_{t} A^{k} Z_{t+i-k} Z_{t+i-k}^{\prime}
$$

Notice that:

$$
\begin{aligned}
(I-\rho A) T_{4 t} & =T_{4 t}-\sum_{i=1}^{\infty} \rho^{i+1} \sum_{k=0}^{i-1} E_{t} A^{k+1} Z_{t+i-k} Z_{t+i-k}^{\prime} \\
& =T_{4 t}-\sum_{i=2}^{\infty} \rho^{i}\left(\sum_{k=0}^{i-1} E_{t} A^{k} Z_{t+i-k} Z_{t+i-k}^{\prime}-E_{t} Z_{t+i} Z_{t+i}^{\prime}\right) \\
& =\sum_{i=1}^{\infty} \rho^{i} E_{t} Z_{t+i} Z_{t+i}^{\prime} \\
& =T_{3 t} .
\end{aligned}
$$

Thus $T_{4 t}=B T_{3 t}$.

The three extra terms $T_{2}, T_{3}$ and $T_{4}$ come as quadratic corrections to the linear measure of $q$. 


\subsection{Standard error bands — univariate case}

This appendix deals with two issues concerning the delta method that underpins the construction of the standard-error bands in subsection 3.2: least-squares small-sample bias and second-order approximation. To this effect, consider the general case of a standard AR(1) process,

$$
x_{t}=\phi x_{t-1}+\varepsilon_{t}
$$

and a process that depends on it in expected present value form:

$$
y_{t}=E_{t} \sum_{i=1}^{\infty} \rho^{i} x_{t+i}=\frac{\rho \phi}{1-\rho \phi} x_{t} \equiv f(\phi) x_{t}
$$

Since $\phi$ needs to be estimated, the observed process for $y$ is $\hat{y}_{t}=f(\hat{\phi}) x_{t}$. Parameter uncertainty means that one can calculate the variance of the expression above conditional on the knowledge of $x$. The standard procedure is to use the first-order delta method, so that

$$
E\left[\left(\hat{y}_{t}-y_{t}\right)^{2} \mid x_{t}\right]=f^{\prime}(\phi)^{2} E\left[(\hat{\phi}-\phi)^{2}\right] x_{t}^{2} .
$$

For practical purposes, the variance of $\hat{\phi}$ is typically used in lieu of the mean square error of $\hat{\phi}$ on the right-hand side of this expression. The two are not the same because of the small-sample bias that affects the least-squares estimation of $\phi$. Moreover, the first-order approximation of $f$ is poor for values of $\phi$ and $\rho$ close to one. The following derivations help quantify the effect of adopting these two simplifications.

Consider the distribution of the least-squares estimator

$$
\hat{\phi} \sim N\left(\bar{\phi}, \frac{1-\phi^{2}}{T}\right)
$$

where $\bar{\phi}=\phi+\theta$, and $\theta$ is the bias. The distribution is asymptotically normal if innovations $\varepsilon$ are normally distributed. It is well-known that to a first order, $\theta \simeq-\frac{2 \phi}{T}$ (see Hendry (1995), for example). The second-order approximation for the measure of dispersion of $\hat{y}$ due to 
parameter uncertainty involves the term

$$
\begin{aligned}
E X & \equiv E[f(\hat{\phi})-f(\phi)]^{2} \\
& =f^{\prime}(\phi)^{2} E\left[(\hat{\phi}-\phi)^{2}\right]+f^{\prime}(\phi) f^{\prime \prime}(\phi) E\left[(\hat{\phi}-\phi)^{3}\right]+\frac{f^{\prime \prime}(\phi)}{4} E\left[(\hat{\phi}-\phi)^{4}\right]
\end{aligned}
$$

Using the decomposition $(\hat{\phi}-\phi)=(\hat{\phi}-\bar{\phi})+(\bar{\phi}-\phi)$, one obtains:

$$
\begin{aligned}
E\left[(\hat{\phi}-\phi)^{2}\right] & =E z^{2}+\theta^{2} \\
E\left[(\hat{\phi}-\phi)^{3}\right] & =E z^{3}+\theta^{3}+3 \theta E z^{2} \\
E\left[(\hat{\phi}-\phi)^{4}\right] & =E z^{4}+\theta^{4}+4 \theta E z^{3}+6 \theta^{2} E z^{2},
\end{aligned}
$$

where $E z^{i}=E\left[(\hat{\phi}-\bar{\phi})^{i}\right]$ is the $i^{\text {th }}$ centered moment. Since $\hat{\phi}$ is normal, the third and fourth moments are zero, so the following terms are left:

$$
\begin{aligned}
& E\left[(\hat{\phi}-\phi)^{2}\right]=E z^{2}+\theta^{2} \sim O\left(T^{-1}\right)+O\left(T^{-1}\right) \\
& E\left[(\hat{\phi}-\phi)^{3}\right]=3 \theta E z^{2}+\theta^{3} \sim O\left(T^{-2}\right)+O\left(T^{-3}\right) \\
& E\left[(\hat{\phi}-\phi)^{4}\right]=6 \theta^{2} E z^{2}+\theta^{4} \sim O\left(T^{-3}\right)+O\left(T^{-4}\right) .
\end{aligned}
$$

Considering those only up to the second order:

$$
\begin{aligned}
E X & =f^{\prime}(\phi)^{2}\left(E z^{2}+\theta^{2}\right)+f^{\prime}(\phi) f^{\prime \prime}(\phi) 3 \theta E z^{2} \\
& =f^{\prime}(\phi)^{2} E z^{2}\left[1+\frac{\theta^{2}}{E z^{2}}+3 \frac{f^{\prime \prime}(\phi)}{f^{\prime}(\phi)} \theta\right]
\end{aligned}
$$

Thus,

$$
E\left[\left(\hat{y}_{t}-y_{t}\right)^{2} \mid x_{t}\right]=f^{\prime}(\phi)^{2} E z^{2}\left[1+\frac{\theta^{2}}{E z^{2}}+3 \frac{f^{\prime \prime}(\phi)}{f^{\prime}(\phi)} \theta\right] x_{t}^{2}
$$

Notice that the first-order approximation of $f(\phi)$ is sufficient only in the absence of bias. The second term in the brackets is a measure of the discrepancy in the construction of standard error bands for $\hat{y}$ that arises from replacing the MSE of $\hat{\phi}$ by its variance. The third term is a measure of the second-order term of the Taylor-expansion, which is negative because of the downward small-sample bias in estimating $\phi$. Replacing the derivatives of $f$ 
and the value of the variance and bias of $\hat{\phi}$ yields

$$
E\left[\left(\hat{y}_{t}-y_{t}\right)^{2} \mid x_{t}\right]=f^{\prime}(\phi)^{2} E z^{2}\left[1+\frac{4}{T}\left(\frac{\phi^{2}}{1-\phi^{2}}-3 \frac{\rho \phi}{1-\rho \phi}\right)\right] x_{t}^{2} .
$$

This expression can be evaluated at $\phi=\hat{\phi}$. For $\rho>\phi$, the correction is quite clearly negative. The error in using $E z^{2}$ and the first order approximation of the delta method instead of the MSE and the second order can be substantial. The values used in subsection 3.2 are $\rho=0.988, \hat{\phi}=0.927$ and $T=200$. This implies that the correct RMSE bands are smaller than standard-error bands implicitly computed by Abel and Blanchard by a factor of 0.72 , at least $25 \%$ tighter.

These derivations were conducted in the case of a univariate process. Clearly, this can be extended to the multivariate case, which is the appropriate one in Abel and Blanchard, but this is left for future work.

\subsection{Standard error bands - VAR case}

The following results are inspired by Abel and Blanchard (1986) once more, but extend their result to account for common parameter restrictions across equations.

Suppose a constructed time-series $\hat{y}_{t}$ can be written as

$$
\hat{y}_{t}=\delta \nu \hat{A} \hat{B} Z_{t}=f(\operatorname{vec}(\hat{A})) Z_{t},
$$

where $\delta$ is a scalar, $\nu$ is a $1 \times n \ell$ vector of known constants, $B \equiv(1-\rho A)^{-1}, \operatorname{vec}(\hat{A})$ is the $n^{2} \ell \times 1$ column vector of the stacked estimated rows of the companion matrix $A$, and $n$ and $\ell$ are the number of variables and lags in the $n$ equations of the VAR, respectively. Differentiating $f$ with respect to any argument $x$ yields

$$
\frac{d f(\operatorname{vec}(\hat{A}))}{d x}=\delta \nu \hat{B} \frac{d \hat{A}}{d x} \hat{B}
$$

Notice that $d \hat{A} / d \hat{a}_{i j}=e_{i}^{\prime} e_{j}$, where the $e_{i}$ vector is the selection vector described in sub-section 3.3 , the $i_{\text {th }}$ row of the identity matrix $I_{n \ell}$. Define $\frac{d f(\operatorname{vec}(\hat{A}))}{d \operatorname{vec}(\hat{A})}$ as the $n^{2} \ell \times n \ell$ matrix of the 
derivatives with respect to each element of $\operatorname{vec}(\hat{A})$ :

$$
\frac{d f(\operatorname{vec}(\hat{A}))^{\prime}}{d \operatorname{vec}(\hat{A})}=\left[\frac{d f(\operatorname{vec}(\hat{A}))}{d \hat{a}_{1,1}}, \ldots, \frac{d f(\operatorname{vec}(\hat{A}))}{d \hat{a}_{1, n \ell}}, \frac{d f(\operatorname{vec}(\hat{A}))}{d \hat{a}_{2,1}}, \ldots, \frac{d f(\operatorname{vec}(\hat{A}))}{d \hat{a}_{n, n \ell}}\right]
$$

It can be written in more compact form:

$$
\frac{d f(\operatorname{vec}(\hat{A}))}{d \operatorname{vec}(\hat{A})}=\delta\left(\left[I_{n} \vdots 0_{n \times n(\ell-1)}\right] \hat{B}^{\prime} \nu^{\prime}\right) \otimes \hat{B}
$$

The purpose of this computation is that the delta method provides a linear approximation of the variance of non-linear functions of parameters:

$$
\operatorname{var}\left(\hat{y}_{t} \mid Z_{t}\right)=Z_{t}^{\prime} \frac{d f(\operatorname{vec}(\hat{A}))^{\prime}}{d \operatorname{vec}(\hat{A})} \operatorname{var}(\operatorname{vec}(\hat{A})) \frac{d f(\operatorname{vec}(\hat{A}))}{d \operatorname{vec}(\hat{A})} Z_{t}
$$

Recalling that $\operatorname{var}(\operatorname{vec}(\hat{A}))=\Omega \otimes\left(Z^{\prime} Z\right)^{-1}$, replacing terms and solving out the Kronecker products yields

$$
\operatorname{var}\left(\hat{y}_{t} \mid Z_{t}\right)=\delta^{2} \nu \hat{B} \Sigma \hat{B}^{\prime} \nu^{\prime} \times Z_{t}^{\prime} \hat{B}^{\prime}\left(Z^{\prime} Z\right)^{-1} \hat{B} Z_{t},
$$

where $\Sigma=\left[\begin{array}{ll}\Omega & 0 \\ 0 & 0\end{array}\right]$.

Now, suppose that the process for $y$ is constructed with the prior knowledge that a variable, say the $j^{\text {th }}$ one, does not Granger-cause the others. That is, its parameters are equal to zero at all lags. Under this maintained hypothesis, since VAR estimation amounts to least square estimation of each individual equation, the rows of the restricted matrix $\tilde{A}$ are the following transformation of the rows of the unrestricted matrix $\hat{A}$ :

$$
\tilde{A}_{i}=\hat{A}_{i} \cdot\left(I_{n \ell}-C^{\prime}\right)
$$

If the restriction is imposed on row $i$, the correction matrix is

$$
C=\left(Z^{\prime} Z\right)^{-1} R^{\prime}\left[R\left(Z^{\prime} Z\right)^{-1} R^{\prime}\right]^{-1} R
$$

where the restriction vector $R=\sum_{k=1}^{\ell} e_{j \times k}^{\prime} e_{j \times k}$ satisfies $R A_{i}^{\prime}$. $=0_{n \ell \times 1}$. Otherwise, $R=C=0$. 
Then

$$
\begin{aligned}
\operatorname{vec}(\tilde{A})= & {\left[\begin{array}{c}
\operatorname{vec}\left(\tilde{A}_{1 .}\right) \\
\vdots \\
\operatorname{vec}\left(\tilde{A}_{n .}\right)
\end{array}\right]=\left[\begin{array}{c}
\operatorname{vec}\left(\hat{A}_{1 \cdot}\left(I_{n}-C^{\prime}\right)\right) \\
\vdots \\
\operatorname{vec}\left(\hat{A}_{n \cdot}\left(I_{n}-C^{\prime}\right)\right)
\end{array}\right] } \\
= & {\left[\begin{array}{ccc}
I_{n}-C & 0 \\
& \ddots & \\
0 & & I_{n}-C
\end{array}\right] \operatorname{vec}(\hat{A})=\operatorname{Mvec}(\hat{A}) . }
\end{aligned}
$$

This implies that

$$
\operatorname{var}(\operatorname{vec}(\tilde{A}))=\operatorname{Mvar}(\operatorname{vec}(\hat{A})) M^{\prime} .
$$

Therefore, by analogy with the derivation above, if the $y$ series is constructed under the maintained hypothesis, one obtains:

$$
\operatorname{var}\left(\hat{y}_{t} \mid Z_{t}, R\right)=Z_{t}^{\prime} \frac{d f(\operatorname{vec}(\tilde{A}))^{\prime}}{d \operatorname{vec}(\tilde{A})} \operatorname{Mvar}(\operatorname{vec}(\hat{A})) M^{\prime} \frac{d f(\operatorname{vec}(\tilde{A}))}{d \operatorname{vec}(\tilde{A})} Z_{t}
$$

Since the restrictions are the same across equations for which they hold, $M$ can be written as

$$
M=I_{n^{2} \ell}-(D \otimes C),
$$

where $D$ is the $n \times n$ identity matrix with zeros on the diagonal where restricted estimation is not required. One can therefore write

$$
\begin{aligned}
M \frac{d f(\operatorname{vec}(\tilde{A}))}{d \operatorname{vec}(\tilde{A})} & =\left(I_{n^{2} \ell}-(D \otimes C)\right)\left(\delta\left([I \vdots 0] \tilde{B}^{\prime} \nu^{\prime}\right) \otimes \tilde{B}\right) \\
& =\delta\left([I \vdots 0] \tilde{B}^{\prime} \nu^{\prime}\right) \otimes \tilde{B}-\delta\left([D \vdots 0] \tilde{B}^{\prime} \nu^{\prime}\right) \otimes C^{\prime} \tilde{B}
\end{aligned}
$$

where now, $\tilde{B}=(I-\rho \tilde{A})^{-1}$. This implies that

$$
\begin{aligned}
\operatorname{var}\left(\hat{y}_{t} \mid Z_{t}, R\right)= & Z_{t}^{\prime}\left[\delta\left([I \vdots 0] \tilde{B}^{\prime} \nu^{\prime}\right) \otimes \tilde{B}-\delta\left(\left[D_{n} \vdots 0\right] \tilde{B}^{\prime} \nu^{\prime}\right) \otimes C^{\prime} \tilde{B}\right]^{\prime} \\
& \left(\Omega \otimes\left(Z^{\prime} Z\right)^{-1}\right)\left[\delta\left([I \vdots 0] \tilde{B}^{\prime} \nu^{\prime}\right) \otimes \tilde{B}-\delta\left(\left[D_{n} \vdots 0\right] \tilde{B}^{\prime} \nu^{\prime}\right) \otimes C^{\prime} \tilde{B}\right] Z_{t}
\end{aligned}
$$

Tediously solving out the Kronecker products while using the fact that $\Omega$ is symmetric, that 
$C$ and $D$ are idempotent, and that

$$
C\left(Z^{\prime} Z\right)^{-1} C^{\prime}=C\left(Z^{\prime} Z\right)^{-1}=\left(Z^{\prime} Z\right)^{-1} C^{\prime} \equiv \Gamma
$$

yields:

$$
\begin{aligned}
\operatorname{var}\left(\hat{y}_{t} \mid Z_{t}, R\right)= & \delta^{2} \nu \tilde{B} \Sigma \tilde{B}^{\prime} \nu^{\prime} \times Z_{t}^{\prime} \tilde{B}^{\prime}\left(Z^{\prime} Z\right)^{-1} \tilde{B} Z_{t} \\
& -\delta^{2} Z_{t}^{\prime}\left(\nu \tilde{B}[I \vdots 0]^{\prime} \Omega[D \vdots 0] \tilde{B}^{\prime} \nu^{\prime} \otimes \tilde{B}^{\prime} \Gamma \tilde{B}\right) Z_{t} \\
= & \delta^{2} \nu \tilde{B} \Sigma \tilde{B}^{\prime} \nu^{\prime} \times Z_{t}^{\prime} \tilde{B}^{\prime}\left(Z^{\prime} Z\right)^{-1} \tilde{B} Z_{t} \\
& -\delta^{2}\left(\nu \tilde{B} \tilde{\Sigma} \tilde{B}^{\prime} \nu^{\prime}\right) \times Z_{t}^{\prime} \tilde{B}^{\prime} \Gamma \tilde{B} Z_{t}
\end{aligned}
$$

where $\tilde{\Sigma}=\left[\begin{array}{cc}D \Omega & 0 \\ 0 & 0\end{array}\right]$. If the restriction is valid to the point where $\hat{A} \simeq \tilde{A}$ and $\hat{B} \simeq \tilde{B}$, then this expression is the system equivalent of the standard result that unrestricted estimated parameter variances differ from the restricted ones by a positive semi-definite matrix, implying that precision is improved by extraneous information embodied in the restrictions.

\subsection{Data description}

Labor share: Rotemberg and Woodford (1999) use a measure of the labor share that is different to the unit labor cost series produced by the Bureau of Labor Statistics. Their reasoning is that the concept of income that matters for firms is net revenue, equivalent to gross product conventionally defined minus indirect business taxes. This paper follows their lead, by computing the (log of the) corporate labor share as the ratio of corporate employee compensation, $w+n$ (in logs), to this concept of net income, $p+y$, both of which are available from table T1.16 of the National Income and Product Accounts. This (log-)share is linearly detrended over the sample period under consideration, but this procedure has a limited impact on the results of the paper, since the trend is virtually flat.

average $Q$ : The time-series for average $Q$ is the quarterly ratio of market value $V$ to tangible assets $p_{K} K$ of the non-financial corporate sector, obtainable from the Flow of Funds of the Federal Reserve. Notice that it is a ratio of nominal variables. Robertson and 
Wright (2002a) correct this measure by accounting for net worth. However, to be consistent with the data for the capital-output ratio described below, this paper retains tangible assets for the denominator of $q$. Since both this series and Robertson and Wright's are correlated at $99.3 \%$, this choice does not affect the results much (this has been checked by running the full programme of this paper on both measures).

Output, consumption and hours: The data used for the estimation of the vector autoregressive process is (practically) the same as the one Rotemberg and Woodford (1996) use in their study of forecastable movements in Real Business Cycle models. The output series is real private output. The average propensity to consume is then computed using real consumption of non-durables and services. Price inflation is constructed with the implicit price deflator. The three series are obtained from the National Income and Product Accounts. The $c-y$ series exhibits a puzzling downward trend in the post-war period, and is therefore detrended to make it suitable for VAR estimation. Furthermore, detrended hours $n$ are computed using data from the Bureau of Labor Statistics, and represent seasonally adjusted aggregate weekly hours of all production workers, aggregated per quarter. Because this series is available only from 1964 onwards, prior data is constructed from the BLS series of quarterly percent changes in total hours worked in the non-farm business.

Notice that the output data used for the construction of the first two elements of the information set is not the same as the one used in the construction of the labor share. Although the two series, private and corporate output, are correlated at $90 \%$ in difference form over the sample, the results in this paper are sensitive to the choice of denominator for the labor share and the capital-output ratio. The use of the corporate output series is more intuitive for the construction of the labor share, but less so for that of the average propensity to consume and the discount factor.

Output-capital ratio: The numerator is the same as the denominator of the labor share, while the denominator is the same as the denominator of average $Q$. The ratio is detrended over the period of interest.

Investment-capital ratio: the denominator is the same as the denominator of average $Q$, 
while the numerator is non-residential private fixed investment, available from NIPA Table 5.4. This ratio is also detrended over the period of interest.

\section{References}

Abel, A. B. (1998). Risk premia and term premia in general equilibrium. NBER Working Paper W6683.

Abel, A. B. and Blanchard, O. J. (1986). The present value of profits and cyclical movements in investment, Econometrica 54: 249-273.

Blanchard, O. J., Rhee, C. and Summers, L. (1993). The stock market, profit and investment, Quarterly Journal of Economics 108: 115-136.

Bond, S. R. and Cummins, J. G. (2001). Noisy share prices and the $q$ model of investment. Working Paper W122, Institute of Fiscal Studies, London.

Caballero, R. (1999). Aggregate investment, in J. Taylor and M. Woodford (eds), Handbook of Macroeconomics, Vol. 1B, Elsevier, North-Holland, pp. 813-862.

Campbell, J. Y. and Cochrane, J. (1999). By force of habit: A consumption-based explanation of aggregate stock market behaviour, Journal of Political Economy pp. 205-51.

Campbell, J. Y., Lo, A. W. and Mackinlay, A. C. (1997). The Econometrics of Financial Markets, Princeton University Press, Princeton, NJ.

Campbell, J. Y. and Shiller, R. J. (1988). Stock prices, earnings, and expected dividends, Journal of Finance 43(3): 661-676.

Cochrane, J. (1997). Where is the market going? uncertain facts and novel theories. Economic Perspectives, Federal Reserve Bank of Chicago.

Fazzari, S., Hubbard, R. and Petersen, B. (1988). Financing constraints and corporate investment, Brookings Paper on Economic Activity 1: 141-195.

Hall, R. E. (2001). The stock market and capital accumulation, American Economic Review 91: $1185-1202$. 
Hayashi, F. (1982). Tobin's marginal $q$ and average $q$ : a neoclassical interpretation, Econometrica 50: 213-224.

Hendry, D. F. (1995). Dynamic Econometrics, Oxford University Press, New York, NY.

Kocherlakota, N. R. (1996). The equity premium: It's still a puzzle, Journal of Economic Literature 34: 42-71.

Lafourcade, P. M. (2003a). Asset prices and rents in a GE model with monopolistic competition. unpublished manuscript.

Lafourcade, P. M. (2003b). Estimating the valuation consequences of markup fluctuations. unpublished manuscript.

Robertson, D. and Wright, S. (2002a). What does $q$ predict? unpublished manuscript.

Robertson, D. and Wright, S. (2002b). The good news and the bad news about long-run stock returns. unpublished manuscript.

Rotemberg, J. J. and Woodford, M. (1995). Dynamic general equilibrium models with imperfectly competitive product markets, in T. Cooley (ed.), Frontiers of Business Cycle Research, Princeton University Press, Princeton, NJ, pp. 243-293.

Rotemberg, J. J. and Woodford, M. (1996). Real business-cycle models and the forecastable movements in output, hours, and consumption, American Economic Review 86(1): 7189.

Rotemberg, J. J. and Woodford, M. (1999). The cyclical behaviour of prices and costs, in J. Taylor and M. Woodford (eds), Handbook of Macroeconomics, Vol. 1B, Elsevier, North-Holland, pp. 1051-1135.

Sargent, T. J. and Ljungqvist, L. (2000). Recursive Macroeconomic Theory, first edn, MIT Press, Cambridge, Massachusetts. 